\title{
Carrier photodynamics in 2D perovskites with solution-processed silver and graphene contacts for bendable optoelectronics
}

\author{
Ridwan F. Hossain ${ }^{1,2}{ }^{2}$, Misook Min ${ }^{1}$, Liang-Chieh Ma ${ }^{1}$, Shambhavi R. Sakri ${ }^{1}$ and Anupama B. Kaul ${ }^{1,2 \bowtie ~}$
}

Silver (Ag) and graphene (Gr) inks have been engineered to serve as efficient electrical contacts for solution-processed twodimensional (2D) organo-halide $\left(\mathrm{CH}_{3}\left(\mathrm{CH}_{2}\right)_{3} \mathrm{NH}_{3}\right)_{2}\left(\mathrm{CH}_{3} \mathrm{NH}_{3}\right)_{n-1} \mathrm{~Pb}_{n} \mathrm{I}_{3 n+1}(n=4)$ layered perovskites, where all inkjet-printed heterostructure photodetectors (PDs) were fabricated on polyimide (PI) substrates. To date, limited studies exist that compare multiple contacts to enable high-performance engineered contacts to 2D perovskites. Moreover, of these few reports, such studies have examined contacts deposited using vapor-based techniques that are time-consuming and require expensive, specialized deposition equipment. In this work, we report on the inkjet printed, direct contact study of solution-processed, 2D perovskite-based PDs formed on flexible PI substrates. Solution processing offers a cost-effective, expedient route for inkjet printing Gr and Ag using a dispersion chemistry developed in this work that is compatible with the underlying 2D perovskite layer to construct the PDs. The wavelength $\lambda$-dependent photocurrent $I_{p}$ peaked at $\lambda \sim 630 \mathrm{~nm}$ for both PDs, consistent with the bandgap $E_{g} \sim 1.96 \mathrm{eV}$ for our semiconducting 2D perovskite absorber layer. The external quantum efficiency was determined to be $103 \%$ for Ag-perovskite PDs, where strain-dependent bending tests were also conducted to reveal the opto-mechanical modulation of the photocurrent in our devices.

npj 2D Materials and Applications (2021)5:34; https://doi.org/10.1038/s41699-021-00214-3

\section{INTRODUCTION}

Organic-inorganic hybrid perovskites described by the formula $A B X_{3}\left(A=\mathrm{CH}_{3} \mathrm{NH}_{3}{ }^{+} ; B=\mathrm{Pb}^{2+}\right.$ or $\mathrm{Sn}^{2+}$; and $\left.X=\mathrm{Cl}^{-}, \mathrm{I}^{-}, \mathrm{Br}^{-}\right)$have recently emerged as attractive materials for solar energy conver$\operatorname{sion}^{1-3}$ and various optoelectronic applications ${ }^{4,5}$. These hybrid perovskites have remarkable optoelectronic properties, such as long carrier lifetimes $\left(\sim 10^{2}-10^{3} \mathrm{~ns}\right)$, high absorption coefficient, high light yield ( 9000 photons/MeV), large exciton binding energy ( $360 \mathrm{meV})$, high carrier mobility, a wide spectral absorption range up to $\sim 800 \mathrm{~nm}$, long carrier diffusion lengths $(\sim 1 \mu \mathrm{m})$, and low charge carrier recombination rates ${ }^{6-8}$. Such properties have positioned these materials for various devices, such as solar cells ${ }^{9,10}$, light-emitting diodes ${ }^{11}$, and photodetectors (PDs) ${ }^{12,13}$. In just a few years, researchers have found ways to increase the power conversion efficiency of single junction perovskite solar cells to over $\sim 25.5 \%$, making it the fastest advancing photovoltaic technology to date ${ }^{10}$. Alongside solar cells, PDs have also attracted significant attention and are finding their way into niche applications, such as optical communications for defense, environmental monitoring, chemical/biological sensing, and space exploration ${ }^{14,15}$.

As an optoelectronic device, a PD converts incoming light into a detectable electrical signal through the generation of charge carriers. It is highly desirable that a PD operate at low-power levels, and at the same time exhibit a high photoresponsivity $R$ and detectivity $D$ with a fast response, and a high ratio of photocurrent-to-dark current, i.e., the ON/OFF ratio. The drawback of commercially available PDs based on materials such as $\mathrm{SiC}, \mathrm{Si}$, InGaAs, $\mathrm{ZnO}^{16-18}$ is their relatively narrow spectral response within the ultra-violet, visible, and infrared regimes, respectively. The hybrid two-dimensional (2D) perovskites when used in PDs have advantages over commercially available PDs, since they exhibit a wide dynamic range given their lower defect densities and tunable bandgaps. External quantum efficiency (EQE) also tends to be impressive over a wide spectral regime ${ }^{19}$. For example, 2D $(\mathrm{EDBE}) \mathrm{PbCl}_{4}$ hybrid perovskite $\left(\mathrm{EDBE}=2,2^{\prime}\right.$-(ethylenedioxy)bis (ethylammonium)) has found its way in X-ray detection, likely to replace currently available materials for scintillators, due largely, to the absence of deep traps and a minimal density of shallow trap states within the detector; here thermal artifacts are also nulledout due to the large exciton binding energy of $2 \mathrm{D}(\mathrm{EDBE}) \mathrm{PbCl}_{4}{ }^{20}$. The absorption coefficient of the $2 \mathrm{D}$ perovskites is also found to be very intimately tied to the direct bandgap nature of the electronic transitions occurring in not only $2 \mathrm{D}$ perovskites, but also $3 \mathrm{D}$ perovskites ${ }^{12}$. These characteristics strongly suggest that the 2D perovskites are ideal candidates for PDs, particularly since their stability appears to be superior to that of conventional 3D perovskites ${ }^{12}$.

In this paper, $2 \mathrm{D}\left(\mathrm{CH}_{3}\left(\mathrm{CH}_{2}\right)_{3} \mathrm{NH}_{3}\right)_{2}\left(\mathrm{CH}_{3} \mathrm{NH}_{3}\right)_{n-1} \mathrm{~Pb}_{n} \mathrm{I}_{3 n+1}$ family of perovskite compounds $(n=4)$ was synthesized using the Ruddlesden-Popper (RP) method ${ }^{19,21,22}$, which is one of the most common techniques used for the synthesis of $2 \mathrm{D}$ perovskites. Previously, a homologous series of RP perovskite phases with the generic formula $\left(\mathrm{CH}_{3}\left(\mathrm{CH}_{2}\right)_{3} \mathrm{NH}_{3}\right)_{2}\left(\mathrm{CH}_{3} \mathrm{NH}_{3}\right)_{n-1} \mathrm{~Pb}_{n} \mathrm{l}_{3 n+1} \quad(n=1-7)$ were synthesized by Ruddlesden and Popper ${ }^{19}$ using butylammonium as the organic spacer, where the number of layers was adjusted by the ratio between the lead source, the methylammonium iodide (MAl), and the butylamine precursor solutions. As eluded to earlier, the potential of $2 \mathrm{D}$ perovskites lies in their enormous structural tunability, which allows flexibility to tailor optoelectronic properties toward a desired application ${ }^{23,24}$. For example, Aharon and $\operatorname{Etgar}^{24}$ synthesized highly luminescent 
$\mathrm{MAPbX}_{3}$ quantum dots, where the $E_{g}$ varied from $\sim 1.90$ to $2.26 \mathrm{eV}$ as the cation $X$ was changed from $\mathrm{I}^{-}$to $\mathrm{Br}^{-}$. In particular, $n$, which is the number of octahedra sheets sandwiched between two organic interlayers, strongly determines the major features of the resulting 2D perovskite, such as bandgap, exciton binding energy, and photoluminescence properties ${ }^{23}$.

One of the key parameters to achieving optimal device performance with reference to transduction of incoming lightto-electricity lies in the ability to control charge carrier flow. The electrical contact between the electrode and the active absorber material is known to play a decisive role in influencing carrier transport ${ }^{25}$. However, fundamental challenges related to contacts often limit the ultimate performance and potential of semiconductors broadly speaking for electronics and optoelectronics, and semiconducting $2 \mathrm{D}$ perovskites are no exception. A Schottky barrier $\Phi_{B}$ between the metal and semiconductor often arises, including in transition metal dichalcogenides (TMDCs) based devices ${ }^{26,27}$; the TMDCs are a broader class of inorganic layered materials ${ }^{28-30}$, just like hybrid organo-halide 2D perovskites where a weak van der Waals interaction serves as the glue between layers. Schottky barriers significantly limit the injection of charge carriers from the semiconducting transport channel region to the external circuit. Lin et al. ${ }^{31}$ conducted a carrier transport study in single-crystalline $\mathrm{CH}_{3} \mathrm{NH}_{3} \mathrm{PbBr}_{3}$ perovskites with $\Phi_{B}$ of $\sim 170,380$, and $470 \mathrm{meV}$ for $\mathrm{Au}, \mathrm{Pt}$, and Ti electrodes, respectively, where lowpower sputtering was used for metal contact deposition. Guo et al. ${ }^{32}$ also presented a simulation model of graphene and other metallic van der Waals solids contacting 2D halide perovskite sheets $\left(\mathrm{C}_{4} \mathrm{H}_{9} \mathrm{NH}_{3}\right)_{2} \mathrm{PbX}_{4}(\mathrm{X}=\mathrm{Cl}, \mathrm{Br}$, and I) for field-effect transistors. To date, the few studies that compare multiple contacts to $2 \mathrm{D}$ perovskites have exclusively relied on vapor-based techniques $^{31,32}$. However, vapor-based techniques are very expensive, time-consuming, and require specialized equipment for the metal contact deposition.

In this report, we provide prototypical demonstrations of inkjet printed, solution-processed electrical contacts to inkjet-printed 2D perovskites formed on flexible substrates. Our devices were constructed with the 2D perovskite serving as the optical absorber layer in a PD platform. The metal contacts explored here include inkjet-printed graphene $(\mathrm{Gr})$ with work function $\phi_{\mathrm{Gr}} \sim 4.5 \mathrm{eV}$, and inkjet-printed silver $(\mathrm{Ag})$ with work function $\phi_{\mathrm{Ag}} \sim 4.26 \mathrm{eV}$, where efficient carrier injection from the 2D perovskite to the external contacts at the metal-semiconductor (MS) interface is validated through temperature $T$-dependent and wavelength $\lambda$-dependent optoelectronic transport measurements of the photocurrent $I_{p}$; from these measurements $\Phi_{B}$ was then extracted. Besides material characterization conducted on our $2 \mathrm{D}\left(\mathrm{CH}_{3}\left(\mathrm{CH}_{2}\right)_{3} \mathrm{NH}_{3}\right)_{2}\left(\mathrm{CH}_{3} \mathrm{NH}_{3}\right)_{n-1}$ $\mathrm{Pb}_{n} \mathrm{I}_{3 n+1}(n=4)$ formulations using techniques such as $\mathrm{x}$-ray diffraction (XRD), including at low-grazing angles $\left(\theta=2.5^{\circ}-12^{\circ}\right)$, and scanning electron microscopy (SEM), the device figures of merit, such as $R$ and $D$, ON/OFF ratio, $\boldsymbol{\tau}_{\text {rise }}$ and $\boldsymbol{\tau}_{\text {fall, }}$ EQE were calculated for both the Gr and the Ag-contacted PDs. Additionally, the $\lambda$-dependent $I_{p}$ and strain-dependent bending measurements were also performed. Our promising results, particularly with Ag contacts, confirm the excellent potential of inkjet-printed 2D perovskites for enabling high-performance PDs on flexible substrates using scalable, additive manufacturing production routes for flexible electronics and solar cells in the future.

\section{RESULTS AND DISCUSSION \\ Material analysis}

In order to construct our devices using the additive manufacturing process of inkjet printing, first, the $\mathrm{Gr}$ and $\mathrm{Ag}$ inks were formulated using isopropyl alcohol (IPA, 2-propanol) solvent. This is an atypical choice of solvent for inkjet printing, but this choice was necessitated by the constraints of post-processing the underlying
2D perovskite layer upon which the contact layer was printed. Since the perovskite layer degrades at $T>100^{\circ} \mathrm{C}^{33}$, low-boiling point solvents used to disperse the $2 \mathrm{D}$ metal inks as the contact layer to the underlying perovskite provide the needed advantage during the annealing step to restrict annealing $T$ to $<100^{\circ} \mathrm{C}$. Specifics of the ink formulation steps and the optimized inkjet printing parameters used for printing the perovskite and the contact layers are provided in the "Methods" section. Figure 1 illustrates the complete process flow from a to d. Shown in Fig. 1a, the ink formulation procedure for the 2D perovskite is outlined on the left, while the Gr/Ag ink dispersion chemistry is shown on the right. The inks were formulated using magnetic stirring for the perovskite formulation in dimethylformamide (DMF) (left) and for the $\mathrm{Gr} / \mathrm{Ag}$ formulation was in IPA using ultrasonication (right). The bottom-left inset in Fig. 1a represents the crystalline structure of the synthesized $2 \mathrm{D}$ perovskite $(\mathrm{BA})_{2}(\mathrm{MA})_{3} \mathrm{~Pb}_{4} \mathrm{I}_{13}$, where the $\mathrm{BA}$ represents $n$-butylamine $\left(\mathrm{CH}_{3}\left(\mathrm{CH}_{2}\right)_{3} \mathrm{NH}_{2}\right)$, and $\mathrm{MA}$ is the methylammonium $\left(\mathrm{CH}_{3} \mathrm{NH}_{3}\right)$. The inkjet printing sequence for the heterostructure device architecture is depicted in b, while in c the annealing conditions are shown with the specific temperature constraints. Depicted in Fig. $1 \mathrm{~d}$ is the final inkjet-printed heterostructure device with the $\mathrm{Gr}$-perovskite and Ag-perovskite shown on the top and bottom, respectively. Figure $1 \mathrm{~b}$ shows the construction of the inkjet-printed heterostructure perovskite (yellow dispersion) and the $\mathrm{Gr} / \mathrm{Ag}$ metal contact layer (blue dispersion) devices. Precautionary measures were needed in printing the $\mathrm{Gr} / \mathrm{Ag}$ dispersions in low-boiling point solvents such as IPA, to overcome nozzle clogging issues, where a mandatory nozzle cleaning sequence was necessary since the IPA in the $\mathrm{Gr} /$ $\mathrm{Ag}$ ink dries out quickly. Details on the nozzle cleaning procedure for the $\mathrm{Gr} / \mathrm{Ag}$ ink is explained in greater detail within the "Methods" section. In Fig. 1c, annealing of the perovskite and $\mathrm{Gr}$ / Ag layers was performed in both cases at $100^{\circ} \mathrm{C}$ for $30 \mathrm{~min}$ at ambient, where the perovskite layer was annealed first right after printing and then it was placed back into the platen to print the $\mathrm{Gr} / \mathrm{Ag}$ layers and annealed subsequently. The annealing is critical to drive off entrapped solvents to yield a dense film for realizing high-integrity electronic devices on polyimide (PI) substrates. Our demonstration of successfully printing IPA-based ink dispersions widens the portfolio of other intriguing temperature-sensitive materials that would otherwise be excluded from the portfolio of flexible electronics materials. Finally, Fig. 1d represents the optical micrograph of the inkjet-printed flexible Gr-perovskite and Agperovskite heterostructure PDs on flexible $\mathrm{PI}$ substrates with printed $\mathrm{Gr}$ (top) and Ag (bottom) contacts, with the perovskite layer beneath the metal contacts.

Charge carrier transport in a perovskite PD is closely influenced by the crystallographic structure and morphology of the photoactive absorber layer to determine device performance ${ }^{34}$. Upon the synthesis of the $2 \mathrm{D}\left(\mathrm{CH}_{3}\left(\mathrm{CH}_{2}\right)_{3} \mathrm{NH}_{3}\right)_{2}\left(\mathrm{CH}_{3} \mathrm{NH}_{3}\right)_{3} \mathrm{~Pb}_{4} \mathrm{I}_{13}$ (exact synthesis procedures are outlined in the "Methods" section), XRD of the inkjet-printed perovskite film was performed at room temperature using the Rigaku Ultima III Diffractometer, as shown in Fig. 2a. The sample was a $\sim 15 \mathrm{~mm}^{2}$ perovskite pattern printed on top of the PI substrate, as shown in the top-left inset of Fig. 2a. The XRD technique characteristically captures the features of the unit cell crystalline lattice by revealing the specific reflections for the 2D $\left(\mathrm{CH}_{3}\left(\mathrm{CH}_{2}\right)_{3} \mathrm{NH}_{3}\right)_{2}\left(\mathrm{CH}_{3} \mathrm{NH}_{3}\right)_{3} \mathrm{~Pb}_{4} \mathrm{I}_{13}$ perovskite film formed using the RP synthesis approach ${ }^{9,21}$. The XRD spectra for the 2D perovskite film displays the typical peaks occurring at $2 \theta=13.84^{\circ}$ assigned to the $\left(\mathrm{CH}_{3}\left(\mathrm{CH}_{2}\right)_{3} \mathrm{NH}_{3}\right)_{2}\left(\mathrm{CH}_{3} \mathrm{NH}_{3}\right)_{3} \mathrm{~Pb}_{4} \mathrm{l}_{13}$ (111) crystallographic planes ${ }^{21}$. Additionally, the $2 \theta$ peaks at $27.23^{\circ}$ and $28.31^{\circ}$ are assigned to the $(0 k 0)$ and (202) crystallographic plane, while the (111) and (202) reflections reveal the vertical growth morphology of the $2 \mathrm{D}$ perovskite with respect to the substrate plane $^{22}$. The top-right inset in Fig. 2a shows the low-grazing angle XRD spectra of the same inkjet-printed film taken from $2 \theta=2.5^{\circ}$ up to $12^{\circ}$. The low-grazing angle XRD reveals the number of layers 


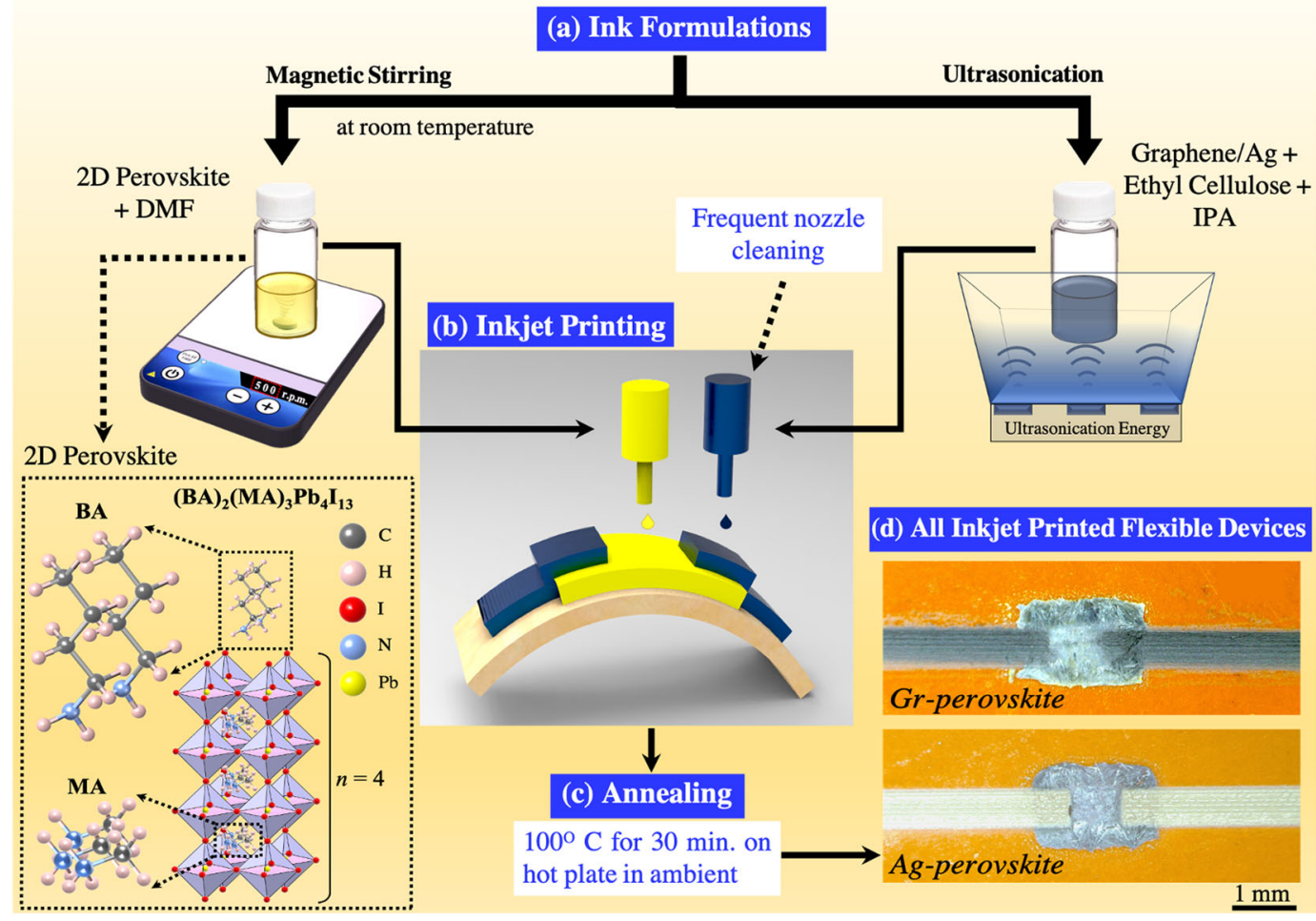

Fig. 1 Complete fabrication process. A complete process flow is shown in this figure that provides a pictorial representation of the ink formulation features in (a), inkjet printing in (b), annealing in (c), and micrographs of fabricated devices in (d). In (a), the perovskite (left) and $\mathrm{Gr} / \mathrm{Ag}$ inks (right) were formulated using magnetic stirring and ultrasonication, respectively. The details of the ink formulations are explained in the "Methods" section. The bottom-left inset in (a) depicts the crystalline structure of $(\mathrm{BA})_{2}(\mathrm{MA})_{3} \mathrm{~Pb}_{4} \mathrm{I}_{13}$, a family of $n=42 \mathrm{D}$ perovskite compounds, where the BA represents $n$-butylamine $\left(\mathrm{CH}_{3}\left(\mathrm{CH}_{2}\right)_{3} \mathrm{NH}_{2}\right)$, and $\mathrm{MA}$ is the methylammonium $\left(\mathrm{CH}_{3} \mathrm{NH}_{3}\right)$. b inkjet printing of the formulated perovskite (yellow ink) and $\mathrm{Gr} / \mathrm{Ag}$ (blue ink), to form our heterostructure flexible PD device. Frequent nozzle cleaning is necessary for the Gr/Ag ink as the IPA present in the Gr/Ag ink dries out fast. c Annealing of the perovskite and Gr/Ag layers was performed in both cases at $100{ }^{\circ} \mathrm{C}$ for $30 \mathrm{~min}$ at ambient, where the perovskite layer was annealed first right after printing and then it was placed back into the platen to print the $\mathrm{Gr} / \mathrm{Ag}$ layers and an additional annealing step was subsequently conducted. d Optical micrograph of the inkjet-printed flexible Grperovskite and Ag-perovskite heterostructure PD devices on flexible PI substrates with printed $\mathrm{Gr}$ (top) and Ag (bottom) contacts, where the metal contacts were printed on top of the underlying 2D perovskite layer.

present in the $2 \mathrm{D}$ perovskite ${ }^{35}$, and is evident from the top-right inset of Fig. 2a, five distinct reflections for $2 \theta<14^{\circ}$ are identified, which occur at $\sim 3.4^{\circ}, 4.5^{\circ}, 6.7^{\circ}, 8.9^{\circ}$, and $10^{\circ}$; this is indicative of the number of $\mathrm{Pbl}_{3}$ octahedra present in each layer of the 2D perovskite crystal. Although our synthesized 2D perovskite was experimentally designed to have four layers (i.e., $n=4$ ) according to the RP synthesis approach, the low-grazing XRD spectra suggests that there may be an additional layer within the compound, which nonetheless is still a $2 \mathrm{D}$ perovskite ${ }^{19,21,35}$, though impurities may also cause this. Ruddlesden and Popper ${ }^{19}$ also indexed an additional peak in their XRD scan for 2D $\left(\mathrm{CH}_{3}\left(\mathrm{CH}_{2}\right)_{3} \mathrm{NH}_{3}\right)_{2}\left(\mathrm{CH}_{3} \mathrm{NH}_{3}\right)_{n-1} \mathrm{~Pb}_{n} \mathrm{I}_{3 n+1} \quad(n=6)$, which included some 3D $n=\infty$ phases and other lower RP phases, whereas in $n=7$, small amounts of $n=5$ and $n=6$ co-crystallize. Nonetheless, the key focus of this paper is the breakthrough studies on solution-processed electrical contacts that are compatible with inkjet-printed heterostructure perovskites for PDs on flexible substrates.

The inkjet-printed films were imaged using an SEM, as shown by the micrographs in Fig. 2b, c, where the heterostructure PDs were printed on a rigid $\mathrm{Si}$ substrate which obviated the need for a conductive coating during SEM imaging. The formation of microfibers within the printed film is clearly evident from this micrograph in Fig. $2 \mathrm{~b}$ and its inset. This characteristic microfibular morphology has been seen in a number of prior studies ${ }^{36-38}$. Wang et al. ${ }^{38}$ reported that a higher $\mathrm{Pbl}_{2}$ to $\mathrm{MAI}$ ratio $\left(>\mathrm{Pbl}_{2}(0.8)\right.$ : $\mathrm{MA}(1)$ ) in the precursor leads to the formation of microfibers within the film, which significantly increases film roughness and yields a very high photoluminescence. In addition, they validated that $\mathrm{MAPbl}_{3}$ resulting from lower $\mathrm{Pbl}_{2}$ to $\mathrm{MAl}$ ratios $\left(<\mathrm{Pbl}_{2}(0.6)\right.$ : MA (1)) do not exhibit the typical photoluminescence and absorption peaks ${ }^{38}$. Figure $2 \mathrm{c}$ illustrates the $52^{\circ}$ tilted top view of the heterostructure PD, where the Gr contacts are apparent on top of the perovskite layer, while its inset on the right displays the magnified view of the printed surface, revealing the embedded network of microfibers and the lateral view of the Gr-perovskite interface.

\section{Electrical transport comparison with $\mathbf{G r}$ and $\mathbf{A g}$ contacts}

After completing the material characterization analysis on the synthesized 2D perovskites and fabricating the inkjet-printed PDs, we then proceeded with the electronic and optoelectronic transport measurements at cryogenic temperatures $T$ from $\sim 4$ to $350 \mathrm{~K}$ using the Lakeshore CRX-4K probe stage and a broadband light source. To compare the $\mathrm{Gr}$ and Ag contacts, a systematic study was performed on the inkjet-printed PDs, where a broadband light source was used to illuminate the devices as they were held in vacuum ( $10^{-6}$ Torr). As mentioned previously, the $\mathrm{Gr}$ and Ag contacts are used in this study and both PDs are fabricated using similar conditions for the ink formulation, printing passes, jetting voltage, and annealing temperature, so that a comparative analysis between the two metal contacts is possible. Figure 3a illustrates the distinct $T$-dependent current $(I)$-voltage $(V)$ Characteristic observed for bias voltages ranging from -40 to $+40 \mathrm{~V}$ at $10.75 \mathrm{~mW} / \mathrm{cm}^{2}$ for the Gr-perovskite PD. On the other hand, 

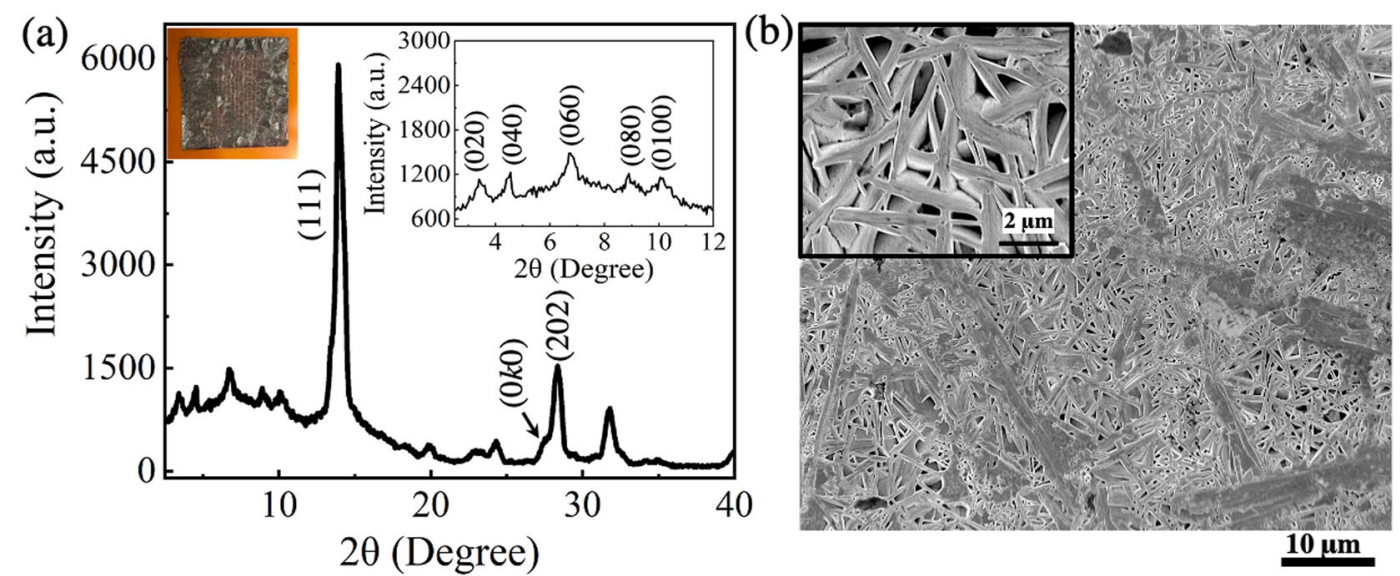

(c)
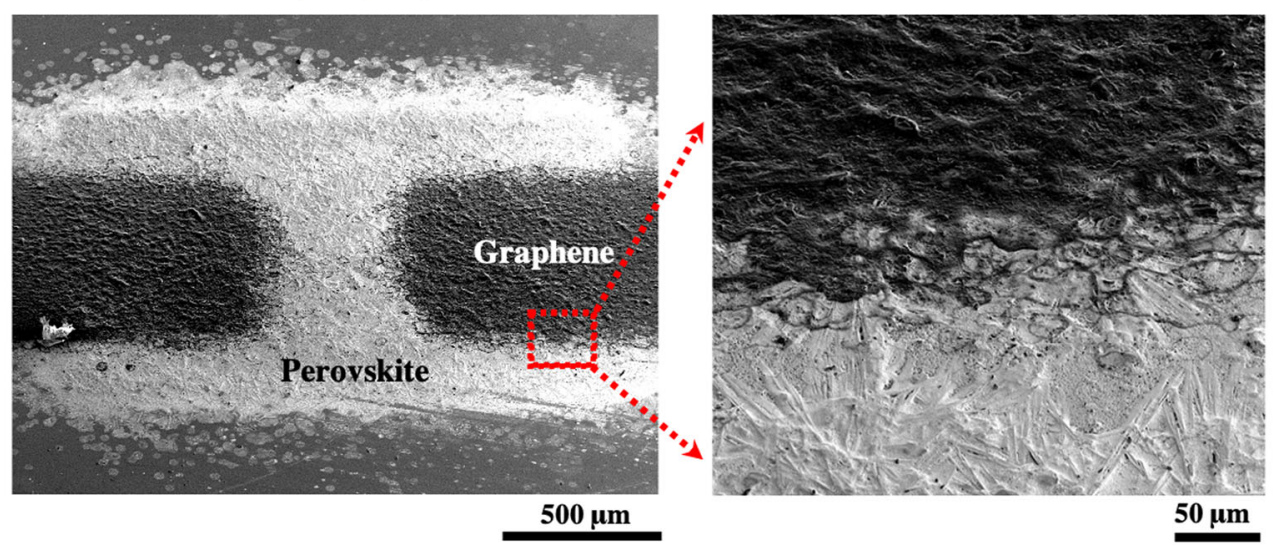

Fig. 2 Material characterization. a The XRD spectra of inkjet-printed $2 \mathrm{D}$ perovskite films on $\mathrm{SiO}_{2} / \mathrm{Si}$ substrates. The $2 \theta$ peaks at $13.84^{\circ}$ and $28.31^{\circ}$ are assigned to the $\left(\mathrm{CH}_{3}\left(\mathrm{CH}_{2}\right)_{3} \mathrm{NH}_{3}\right)_{2}\left(\mathrm{CH}_{3} \mathrm{NH}_{3}\right)_{3} \mathrm{~Pb}_{4} \mathrm{I}_{13}(111)$ and (202) crystallographic planes, respectively. The top-right inset shows lowgrazing angle XRD data, that depicts five distinct reflections at $3.4^{\circ}, 4.5^{\circ}, 6.7^{\circ}, 8.9^{\circ}$, and $10^{\circ}$ for $2 \theta<14^{\circ}$ which is indicative of the number of 2D perovskite layers in the compound. The top-left inset displays the optical microscopy image of the printed perovskite sample on top of the PI substrate over an area $\sim 15 \mathrm{~mm}^{2}$ used for the XRD analysis. b SEM image of an inkjet-printed perovskite heterostructure PD on a Si substrate. The inset shows the magnified view of the microfibular structure of the inkjet-printed 2D perovskites structure, which significantly increases film roughness and yields a very high photoluminescence ${ }^{38}$. c SEM image at $52^{\circ}$ tilt angle of the printed Gr-perovskite PD on a Si substrate, where the right inset displays the Gr-perovskite lateral MS junction.

Fig. $3 b$ displays the $T$-dependent $I-V$ for the Ag-perovskite PD at similar conditions. For both PDs, some nonlinearity in the $I-V$ was observed for $T$ ranging from $\sim 4$ to $300 \mathrm{~K}$, and the response appeared to be more Ohmic at $T \sim 350 \mathrm{~K}$. A sudden rise in the photocurrent $I_{p}$ was observed with respect to $T$, for both PDs and the results are plotted in Fig. $3 c$, showing the $I_{p}$ is increasing as $T$ increases at a fixed light intensity $F$ of $\sim 10.75 \mathrm{~mW} / \mathrm{cm}^{2}$. At low $T$, the electrons are localized within their atomic cores, and as $T$ increases, they gain sufficient energy to move from the valence band into the conduction band, forcing $I_{p}$ to increase as $T$ increases. In Fig. $3 c$, the $I_{p}$ rises with $T$ from $\sim 4$ to $350 \mathrm{~K}$, particularly at higher $T$, for both PDs at a fixed bias voltage of $\sim 40 \mathrm{~V}$. While printing both PDs, the number of printing passes and distance between the metal electrodes were kept constant, so relative comparisons in $I_{p}$ can be made. At $4 \mathrm{~K}, I_{p(4 \mathrm{~K})} \sim 4.0$ and $3.4 \mathrm{nA}$, while at $300 \mathrm{~K}, I_{p(300 \mathrm{~K})} \sim 14$ and $10 \mathrm{nA}$, for Ag-perovskite and Grperovskite, respectively, as summarized in Table 1 . The dark current $I_{d}$ was found to be $\sim 1 \mathrm{pA}$ for both PDs and was $T$ independent, which was measured by turning the broadband light source off. The inset in Fig. $3 c$ shows the $I_{p}$ behavior over a narrower range from $\sim 4$ up to $200 \mathrm{~K}$. At $T>4 \mathrm{~K}$ and $T>50 \mathrm{~K}$, the $I_{p}$ started decreasing until reaching the transition temperature $T_{\text {transition }} \sim 150$ and $170 \mathrm{~K}$ for Ag-perovskite and $\mathrm{Gr}$-perovskite PDs, respectively; the $I_{p}$ started to increase rapidly beyond $T_{\text {transation. }}$ This dip at $T_{\text {transation }}$ has also been observed in prior reports, where the orthorhombic-to-tetragonal phase transition occurring in 2D perovskites between $\sim 150$ and $200 \mathrm{~K}$ is noted as the primary cause ${ }^{39}$. Incidentally, Weller et al. ${ }^{39}$ established that in $\mathrm{CH}_{3} \mathrm{NH}_{3} \mathrm{Pbl}_{3}$, a low- $T$ orthorhombic-to-tetragonal phase transition is evident at $\sim 165 \mathrm{~K}$, whereas the high- $T$ transition from tetragonal-to-cubic takes place at $\sim 327 \mathrm{~K}$. In the orthorhombic phase $(T \sim 4 \mathrm{~K}<T<170 \mathrm{~K})$, where all of the lattice parameters $a, b$, and $c$ are unequal $(a \neq b \neq c)$, a dimensional stress in the unit cell is induced as it transitions to the tetragonal phase, where two of the lattice parameters $a$ and $b$ are equivalent $(a=b \neq c)$. From the $T$ dependent $I-V$ of both PDs, the devices contacted with the Ag electrodes clearly showed $I_{p}$ to be greater for $T>250 \mathrm{~K}$ due to the increased collection of photocarriers compared to the $\mathrm{Gr}$ contacts. This also supports the Schottky barrier analysis data, which is discussed shortly in reference to Fig. 3f.

To compare the charge transport characteristics of our 2D perovskite with $\mathrm{Gr}$ and $\mathrm{Ag}$ contacts, the Schottky barrier height $\Phi_{B}$ was calculated using $T$-dependent transport data. Figure $3 \mathrm{~d}$, e shows the $\ln \left(I_{p} / T^{3 / 2}\right)$ versus $1000 / T$ Arrhenius plot for various $V$ ranging from 0.4 to $4 \mathrm{~V}$ for the $\mathrm{Gr}$-perovskite and Ag-perovskite PDs, respectively. The $\Phi_{B}$ was extracted from the forward-bias fit for $T \sim 50-300 \mathrm{~K}$ using the ideal Schottky diode formula:

$I_{f}=I_{s} \exp ^{q V_{f} / k_{B} T}$

where $I_{f}$ is defined as forward-bias current, and $I_{s}$ the saturation 

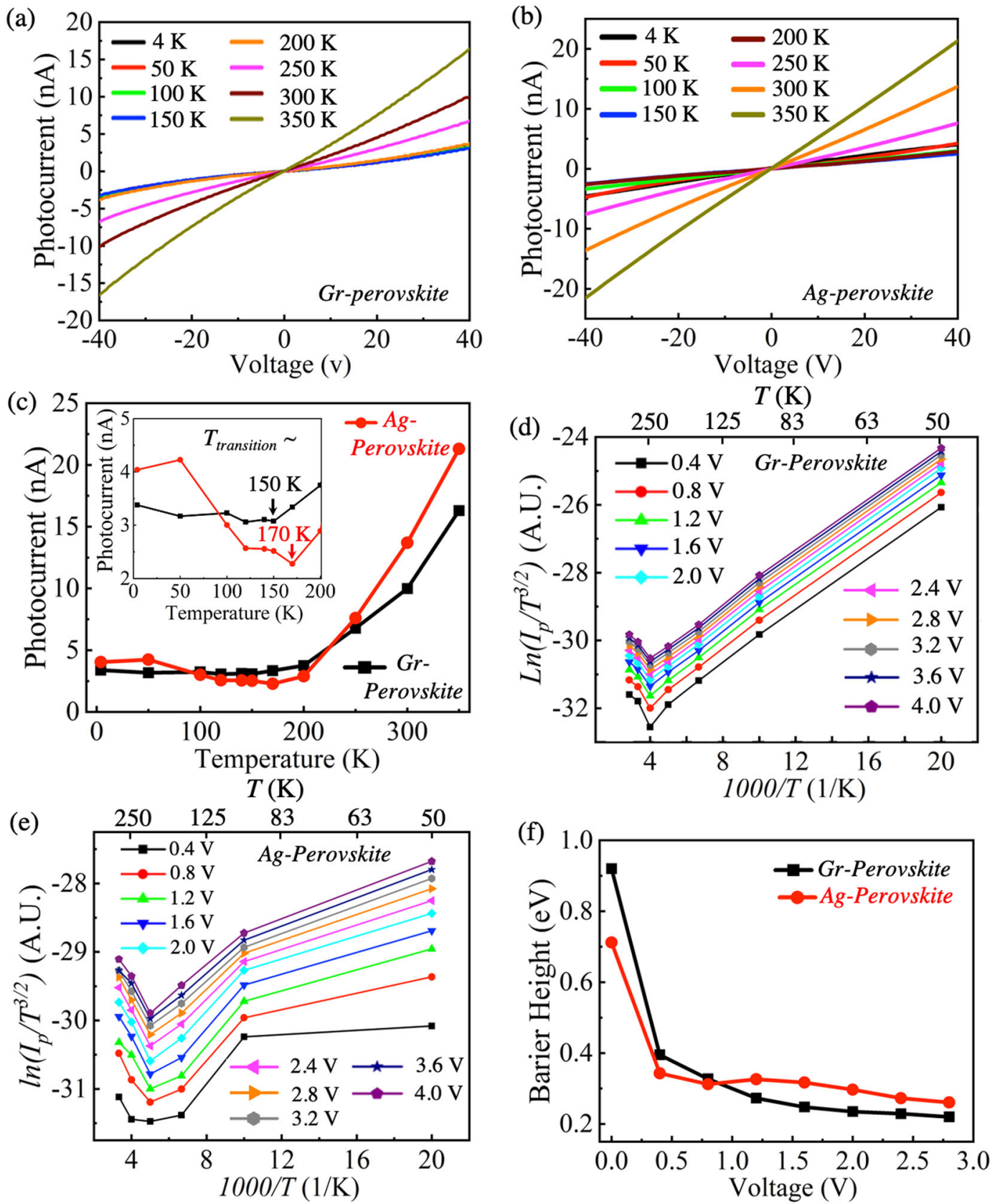

Fig. 3 Electronic and optoelectronic transport data of the 2D perovskite PD. The I-V characteristics of a Gr-perovskite and $\mathbf{b}$ Ag-perovskite heterostructure PDs with $T$ ranging from $\sim 4$ to $350 \mathrm{~K}$ at $F \sim 10.75 \mathrm{~mW} / \mathrm{cm}^{2}$. c The $T$-dependent $I_{p}$ change of Gr-perovskite and Ag-perovskite PDs for $T$ ranging from $\sim 4$ to $350 \mathrm{~K}$. The inset displays the $I_{p}$ behavior for $T$ ranging from $\sim 4$ to $200 \mathrm{~K}$, where the $T_{\text {transition }}$ was observed at $\sim 150$ and $170 \mathrm{~K}$ for Gr-perovskite and Ag-perovskite PDs, respectively, which is likely due to the orthorhombic-to-tetragonal phase transition occurring within the perovskite. The Arrhenius plot of $\ln \left(I_{p} / T^{3 / 2}\right)$ versus $1000 / T$ at bias voltages ranging from 0.4 to $4 \mathrm{~V}$ for $\mathbf{d} \mathrm{Gr}$-perovskite and e Ag-perovskite PDs. f The Schottky barrier height $\Phi_{B}$ as a function of voltage for the Gr-perovskite and Ag-perovskite PDs. At 0 V, the $\Phi_{B}$ was found to be $\sim 920$ and $712 \mathrm{meV}$ for Gr-perovskite and Ag-perovskite PDs, respectively, in the $T$ regime from $\sim 100$ to $150 \mathrm{~K}$.

current that is given by:

$I_{S}=A \omega T^{2} \exp ^{-q \Phi_{B} / k_{B} T}$

here $A$ is the Richardson constant, $\omega$ is the contact area of the metal-perovskite junction, $q$ is the electron charge $(\sim 1.602 \times$ $\left.10^{-19} \mathrm{C}\right), V_{f}$ is the forward-bias voltage, and $k_{B}$ is the Boltzmann constant $\left(\sim 8.617 \times 10^{-5} \mathrm{eV} / \mathrm{K}\right)$. Since our $2 \mathrm{D}$ perovskite falls in the 2D materials family, the $I_{p}$ is defined by the $2 \mathrm{D}$ thermionic emission equation below ${ }^{40}$, which employs the reduced power law for a 2D transport channel according to:

$$
I_{p}=A^{*} \omega T^{\frac{3}{2}} \exp \left[-\frac{q}{k_{B} T}\left(\Phi_{B}-{ }_{n}^{V}\right)\right]
$$

here $A^{*}$ is the 2D equivalent Richardson constant, $n$ is the ideality factor, and $V$ is the bias voltage. The $\Phi_{B}$ was extracted from the $\ln \left(I_{p} / T^{3 / 2}\right)$ versus $1000 / T$ Arrhenius plot in the $T$ regime from $\sim 100$ to $150 \mathrm{~K}$ through the linear fit $y=a+b x$, where $b$ is the slope and $a$ is the $y$-intercept. Figure $3 f$ displays the change in $\Phi_{B}$ with increasing bias voltage. It is observed that at $0.4 \mathrm{~V}$, the $\Phi_{B}$ decreased more than half of its initial value at 0 bias voltage and 
Table 1. Comparison of photocurrent $l_{p}$, barrier height $\Phi_{B}$, photoresponsivity $R$, detectivity $D$, rise time $\boldsymbol{\tau}_{\text {rise }}$ and fall time $\boldsymbol{\tau}_{\text {fall, }}$ ON-OFF ratio, and external quantum efficiency (EQE) of Gr-perovskite and Ag-perovskite flexible PDs at room $T$.

\begin{tabular}{|c|c|c|c|c|c|c|c|c|}
\hline Device architecture & $I_{p}(\mathrm{nA})$ & $\Phi_{B}(\mathrm{meV})$ & $R(\mathrm{~A} / \mathrm{W})$ & $D$ (Jones) & $\tau_{\text {rise, }} \tau_{\text {fall }}(\mathrm{ms})$ & ON-OFF ratio & EQE (\%) & References \\
\hline $\mathrm{Ag}-(\mathrm{BA})_{2}(\mathrm{MA})_{3} \mathrm{~Pb}_{4} \mathrm{I}_{13}$ & 0.4 at $1 \mathrm{~V}$ & 712 & 0.53 & $3.24 \times 10^{13}$ & 17,45 & $3.4 \times 10^{3}$ & 103 & This work \\
\hline $\mathrm{Pt} / \mathrm{CH}_{3} \mathrm{NH}_{3} \mathrm{PbBr}_{3}$ & & 380 & & & & & & \\
\hline $\mathrm{Ti} / \mathrm{CH}_{3} \mathrm{NH}_{3} \mathrm{PbBr}_{3}$ & & 470 & & & & & & \\
\hline $\mathrm{Au} / \mathrm{CsPbBr}_{3}$ & 1.1 at $1 \mathrm{~V}$ & & & & $17.8,14.7$ & 100 & & 51 \\
\hline polycrystalline $\alpha-\mathrm{FAPbl}_{3}$ & & & 0.95 & $2.8 \times 10^{12}$ & $12.4,17.8$ & $8.6 \times 10^{4}$ & 182 & 52 \\
\hline
\end{tabular}

almost saturates above $1 \mathrm{~V}$ for both PDs. At $0 \mathrm{~V}$, the $\Phi_{B}$ was found to be $\sim 920$ and $712 \mathrm{meV}$ for Gr-perovskite and Ag-perovskite, respectively, in the $T$ regime from $\sim 50$ to $100 \mathrm{~K}$. Table 1 summarizes the $\Phi_{B}$ of our inkjet-printed perovskite with $\mathrm{Gr}$ and $\mathrm{Ag}$ contacts. For our synthesized perovskite, the experimental value of $\Phi_{B}$ was compared with the theoretical equivalent, where $\Phi_{B}$ (theoretical) was determined using:

$\Phi_{B}($ theoretical $)=\phi_{\text {metal }}-X$

The calculated theoretical $\Phi_{B}$ values were determined to be $\sim 680$ and $440 \mathrm{meV}$ for $\mathrm{Gr}$-perovskite and Ag-perovskite interfaces, respectively, where the work function $\phi$ of $\mathrm{Gr}$ and $\mathrm{Ag}$ are $\phi_{\mathrm{Gr}}=$ $4.5 \mathrm{eV}$ and $\phi_{\mathrm{Ag}}=4.26 \mathrm{eV}$, respectively, and the perovskite electron affinity $X \sim 3.82 \mathrm{eV}^{41}$. The difference between the theoretical $\Phi_{B}$ of the two contact metals is $\Delta_{\Phi_{B} \text { (theoretical) }}=\Phi_{B(\mathrm{Gr})}-\Phi_{B(\mathrm{Ag})}=680-$ $440 \mathrm{meV}=240 \mathrm{meV}$, whereas at a $0 \mathrm{~V}$ bias voltage, the difference between the experimental $\Phi_{B}$ values is $\Delta_{\Phi_{B} \text { (experimental) }}=\Phi_{B(\mathrm{Gr})}-$ $\Phi_{B} \quad(\mathrm{Ag})=920-712 \mathrm{meV}=208 \mathrm{meV}$, which closely matches $\Delta_{\Phi_{B} \text { (theoretical). }}$ In both the theoretical and experimental cases, $\Phi_{B}$ for Ag was found to be lower than that of the Gr contact. This is in alignment with the higher $I_{p}$ observed in our Ag-contacted 2D perovskite devices, in contrast to the $\mathrm{Gr}$ contacted devices. Limited prior studies exist on contacts to $2 \mathrm{D}$ perovskites using evaporation-assisted metal contact deposition ${ }^{31}$, where $\Phi_{B}$ was found to be $\sim 170,380$, and $470 \mathrm{meV}$ for $\mathrm{Au}, \mathrm{Pt}$, and Ti electrodes, respectively. Our all inkjet-printed electrical contacts yield a facile, reliable, and more cost-effective route to form electrical contacts to $2 \mathrm{D}$ perovskites, which resulted in $\Phi_{B} \sim 712 \mathrm{meV}$ for the MS junction interface with Ag-perovskite. This work shows the important steps toward the analysis of the Schottky barrier between inkjet-printed $\mathrm{Gr}$ and $\mathrm{Ag}$ contacts to 2D perovskites, which should pave the way for their future applications in optoelectronics and photovoltaics.

To fully characterize the performance of our inkjet-printed PDs, their figures of merit were computed, such as $R, D, E Q E, O N / O F F$ ratio, and wavelength $\lambda$-dependent transport, as shown by the data in Fig. 4a-d. Figure 4a provides the dependence of $R$ as a function of light intensity $F$ for both $\mathrm{Gr}$ - and Ag-contacted PDs at $T \sim 300 \mathrm{~K}$ and biased at $40 \mathrm{~V}$. Here, the $R$ is determined using:

$R=\frac{J_{p h}}{F}$

with $J_{p h}$ as the photocurrent density in $\mathrm{A} / \mathrm{cm}^{2}$ and $F$ as the light intensity in $W / \mathrm{cm}^{2}$. From Fig. $4 \mathrm{a}$, the $R$ was calculated to be $\sim 0.27$ and $0.53 \mathrm{~A} / \mathrm{W}$ for the Gr-perovskite and Ag-perovskite PDs, respectively, at $F \sim 5.24 \mu \mathrm{W} / \mathrm{cm}^{2}\left(0.00524 \mathrm{~mW} / \mathrm{cm}^{2}\right)$ and a decrease is noted at higher $F$ for both PDs. Similarly, at $T \sim 300 \mathrm{~K}$, the inset in Fig. 4a shows the corresponding $D$ as a function of $F$ for both PDs at $40 \mathrm{~V}$, where the $D$ is tabulated using:

$D=\frac{\omega^{1 / 2} R}{\left(2 q l_{d}\right)^{1 / 2}}$

here $D$ is measured in $\mathrm{cm} \mathrm{Hz}^{1 / 2} \mathrm{~W}^{-1}$ (Jones), $\omega$ is the effective area $\left(\sim 0.0048 \mathrm{~cm}^{2}\right)$ between the metal contacts, $R$ is the responsivity, and $I_{d}$ is the dark current $(\sim 4 \mathrm{pA})$ in Amps. At $300 \mathrm{~K}$ and $F \sim 5.24 \mu \mathrm{W} / \mathrm{cm}^{2}\left(0.00524 \mathrm{~mW} / \mathrm{cm}^{2}\right), D$ was calculated to be $\sim 1.55 \times 10^{13}$ Jones and $3.24 \times 10^{13}$ Jones for the Gr-perovskite and Ag-perovskite PDs, respectively. It is seen that for the $\mathrm{Ag}$ perovskite PD, both the $R$ and $D$ are $2 \mathrm{X}$ higher compared to the Gr-perovskite PDs. Table 1 summarizes the $R$ and $D$ of our inkjetprinted perovskite with $\mathrm{Gr}$ and $\mathrm{Ag}$ contacts. The $R$ for $\mathrm{Ag}$ perovskite is higher compared to other heterostructure PDs, where the perovskite was contacted with thermally evaporated $\mathrm{Au}$ and ITO ${ }^{42,43}$. In particular, the $\mathrm{Au} / \mathrm{CH}_{3} \mathrm{NH}_{3} \mathrm{Pbl}_{3} / \mathrm{ITO}^{44}$ and $\mathrm{CH}_{3} \mathrm{NH}_{3} \mathrm{Pbl}_{3} / \mathrm{Au}^{42}$ PDs showed $R \sim 0.036$ and $0.037 \mathrm{~A} / \mathrm{W}$, respectively, whereas $\mathrm{Au} / \mathrm{CH}_{3} \mathrm{NH}_{3} \mathrm{Pbl}_{3}$ nanonet $\mathrm{PD}$ demonstrated higher $R \sim 10.33 \mathrm{~A} / \mathrm{W}^{43}$; interestingly the $\mathrm{CH}_{3} \mathrm{NH}_{3} \mathrm{Pbl}_{3}$ nanonets were created by using monolayer colloidal crystal templates. Additionally, 3D perovskites with Au contacts ${ }^{45,46}$ also showed lower $R$ and $D$ compared to our Ag-perovskite PDs. Other combinations of electrode-absorber heterostructures include $\mathrm{Pt} / \mathrm{MAPbCl}_{3} / \mathrm{Ti} / \mathrm{Au}^{47}$ and $\mathrm{Au} / \mathrm{MAPbl}_{3} \mathrm{PDs}^{45}$, which display lower $R \sim 0.0469$ and $0.10 \mathrm{~A} /$ $\mathrm{W}$, respectively, while the $\mathrm{Pt} / \mathrm{MAPbCl}_{3} / \mathrm{Ti} / \mathrm{Au}^{46}$ and $\mathrm{Au} / \mathrm{MAPbl}_{3}{ }^{47}$ showed $10^{3} \times$ lower values of $D$ compared to the Ag-perovskite PD demonstrated here.

Figure $4 \mathrm{~b}$ displays the EQE obtained for both PDs as a function of $T$ at $F \sim 5.24 \mu \mathrm{W} / \mathrm{cm}^{2}\left(0.00524 \mathrm{~mW} / \mathrm{cm}^{2}\right)$. The EQE is defined as the number of electron-hole pairs generated for an incident photon impinging onto the PD, and takes the following form:

$\mathrm{EQE}=R \frac{h c}{\lambda q}$

where $h \sim 6.6 \times 10^{-34} \mathrm{Js}$ is Planck's constant, and all the other variables are as denoted previously with $\lambda \sim 630 \mathrm{~nm}$ for the laser source used. The EQE seems to increase with increasing $T$ for both PDs, reaching $\sim 78 \%$ and $103 \%$ at $\sim 300 \mathrm{~K}$ for the Gr-perovskite and Ag-perovskite, respectively. High EQE values have previously been reported $^{48-50}$ for $\mathrm{WSe}_{2}$, graphene/Si, and GeS-based PDs. The situation of EQE exceeding $100 \%$ arises as charge traps localize the photogenerated carriers, increasing their lifetime and results in their recirculation multiple times before recombination. With this gain mechanism, a higher responsivity and EQE often result ${ }^{50}$. The inset in Fig. $4 b$ shows the EQE as a function of $F$, where the EQE decreases with increasing $F$. At $F \sim 5.24 \mu \mathrm{W} / \mathrm{cm}^{2}(\sim 0.00524 \mathrm{~mW} /$ $\mathrm{cm}^{2}$ ), the $\mathrm{EQE} \sim 103 \%$, whereas at $F \sim 10.75 \mathrm{~mW} / \mathrm{cm}^{2}$, the EQE decreased dramatically to $\sim 0.05 \%$ for the Ag-perovskite PD. Figure $4 \mathrm{C}$ displays the ON/OFF ratio for the Gr-perovskite and Ag-perovskite 
(a)

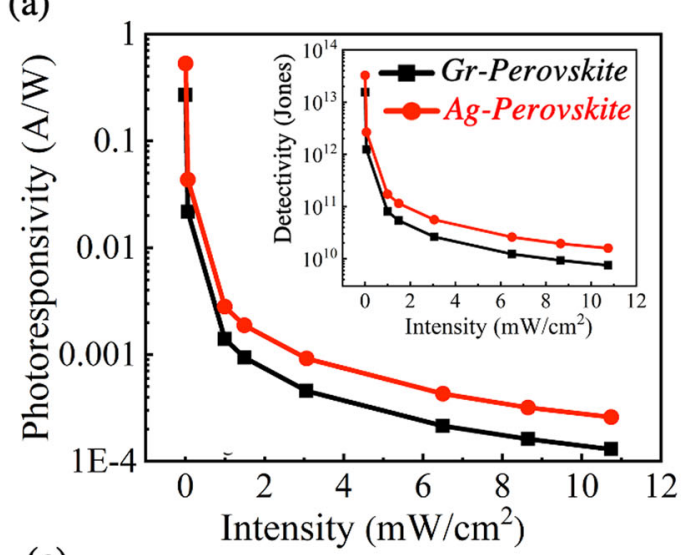

(c)

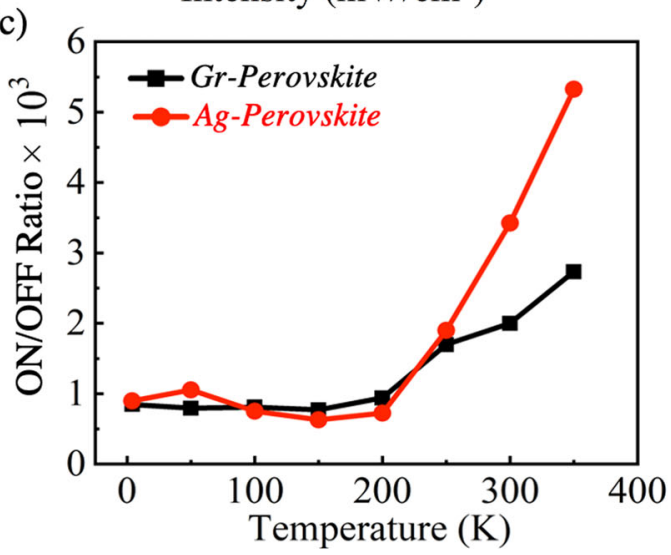

(b)

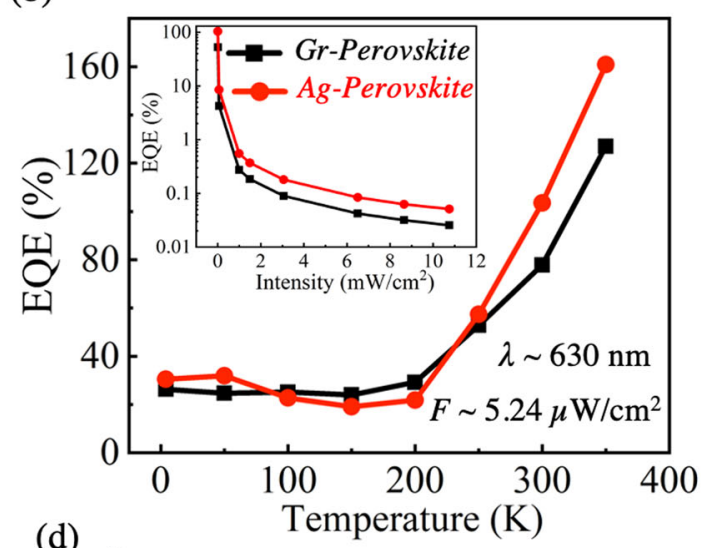

(d)

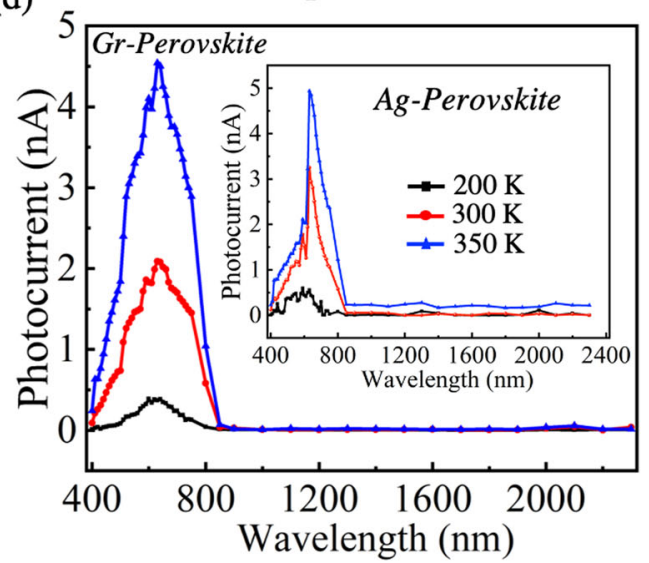

Fig. 4 Device figures of merit for the 2D perovskite PD. a $R$ as a function of $F$, where the highest $R$ was found to be $\sim 0.27$ and $0.53 \mathrm{~A} / \mathrm{W}$ at $F \sim$ $5.24 \mu \mathrm{W} / \mathrm{cm}^{2}\left(0.00524 \mathrm{~mW} / \mathrm{cm}^{2}\right)$ for Gr-perovskite and Ag-perovskite PDs, respectively. The inset shows $D$ as a function of $F$, where $D$ was calculated to be $\sim 1.55 \times 10^{13}$ Jones and $3.24 \times 10^{13}$ Jones for Gr-perovskite and Ag-perovskite, respectively. $\mathbf{b}$ The EQE as a function of $T$ ( 4-350 K). The EQE increases considerably with increasing $T$, and at room temperature, EQE was determined to be $\sim 78 \%$ and $103 \%$ for the Grperovskite and Ag-perovskite PDs, respectively. The inset shows the EQE as a function of $F$ for both PDs. c ON/OFF ratio comparison for both PDs at $T \sim 4-350 \mathrm{~K}$. At $\sim 300 \mathrm{~K}$, the ON/OFF ratios were $\sim 2 \times 10^{3}$ and $3.4 \times 10^{3}$ for Gr-perovskite and Ag-perovskite PDs, respectively. d The $I_{p}$ as a function of $\lambda$ for the Gr-perovskite PD, while the inset shows the corresponding $I_{p}$ versus $\lambda$ for the Ag-Perovskite PD at $T \sim 200,300$, and $350 \mathrm{~K}$.

PDs for $T$ ranging from $\sim 4$ to $350 \mathrm{~K}$, which establishes an important figure-of-merit that is defined as the "ON" state referring to the collected current under illumination, and the "OFF" state referring to the current in the dark. As seen from Fig. 4c, the "ON/OFF" ratio increased with increasing $T$ for both PDs, although at $T \sim 4-170 \mathrm{~K}$, the ON/OFF ratio decreased due to the orthorhombic-to-tetragonal phase transition explained in reference to the data in Fig. 3c. At $\sim 300 \mathrm{~K}$, the ON/OFF ratios were found to be $\sim 2 \times 10^{3}$ and $3.4 \times 10^{3}$ for the Gr-perovskite and Ag-perovskite PDs, respectively, which supports our previous results on the lower Schottky barrier height calculated for the $\mathrm{Ag}$ contacts. Table 1 summarizes the ON/OFF ratios of our inkjetprinted perovskite with $\mathrm{Gr}$ and Ag contacts. The high ON/OFF ratios of $\sim 3.4 \times 10^{3}$ obtained for the Ag-perovskite PDs are at least $\sim 30 \mathrm{X}$ higher compared to prior $\mathrm{PD}$ reports based on other perovskites, such as $\mathrm{CH}_{3} \mathrm{NH}_{3} \mathrm{Pbl}_{3}{ }^{6,47}$ and $\mathrm{CsPbBr}_{3}{ }^{51}$, where the ON/ OFF ratios reported were $\sim 10^{2}$. In yet other similar studies, where polycrystalline a-FAPbl ${ }_{3}^{52}$ were used to fabricate PDs, the ON/OFF ratios obtained were somewhat higher $\sim 8.6 \times 10^{4}$.

We also conducted additional optoelectronic measurements on our PD devices using a tunable fiber-coupled laser source from NKT Photonics (part \#: A371-200-000 and A371-500-000), where $\lambda$ was varied from 400 to $2300 \mathrm{~nm}$. The filters were switched between the measurements due to the restriction in ranges
(400-1000 nm, Filter- M000010357; 1000-2300 nm, FilterM000010266). Figure $4 \mathrm{~d}$ exhibits the $I_{p}$ versus $\lambda$ plot for the $\mathrm{Gr}$ perovskite PD at $T \sim 200,300$, and $350 \mathrm{~K}$, while the inset shows the corresponding $I_{p}$ versus $\lambda$ for the Ag-perovskite PD, where the measurements were made in vacuum at a bias of $40 \mathrm{~V}$. In both cases, the maximum $I_{p}$ was found at $\lambda \sim 630 \mathrm{~nm}$, which is consistent with the bandgap $E_{g} \sim 1.96 \mathrm{eV}$ for our 2D perovskite material. The $E_{g}$ value obtained closely matches the data from prior reports on $2 \mathrm{D}(\mathrm{BA})_{2}(\mathrm{MA})_{3} \mathrm{~Pb}_{4} \mathrm{l}_{13}$, where $E_{g} \sim 1.91 \mathrm{eV}$ was reported $^{21}$, which suggests that the photons with energy $<1.96 \mathrm{eV}$ will not cause optical excitation and thus there is little absorption within the PD devices. Although our synthesized 2D perovskite showed absorption arising over a relatively broad range from $\lambda \sim$ 400 to $880 \mathrm{~nm}$, the $I_{p}$ was least for $\lambda>850 \mathrm{~nm}$, given the maximal $\lambda$ range measured was $2300 \mathrm{~nm}$. Apart from the photon energy of $1.96 \mathrm{eV}$, this $I_{p}$ reduction may also be due to defects and trap states within the layers, which annihilate the generated electron-hole pairs due to non-radiative decay mechanisms.

Figure $5 \mathrm{a}, \mathrm{b}$ displays the temporal response of $I_{p}$ to ON/OFF optical radiation pulses at $T \sim 100,200,300$, and $350 \mathrm{~K}$ with $F \sim$ $10.75 \mathrm{~mW} / \mathrm{cm}^{2}$ for the Gr-perovskite and Ag-perovskite PDs, respectively, where the "ON" pulses are shaded in the first two cycles, as examples. The ON/OFF cycling measurements were conducted using a semiconductor parameter analyzer B1500A and 

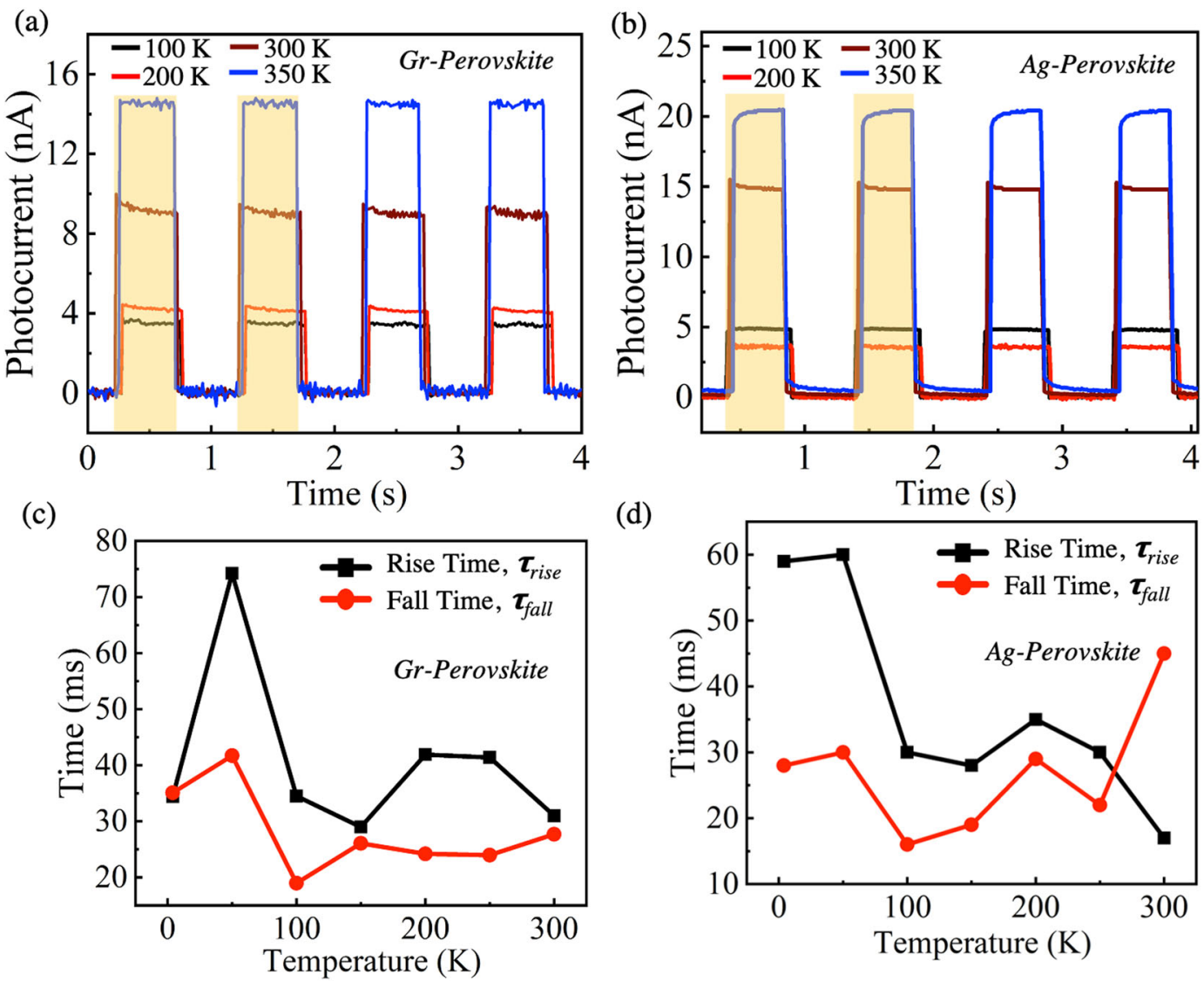

(d)

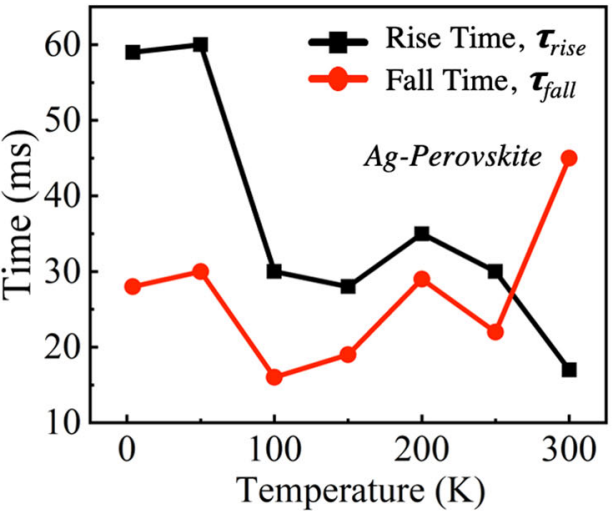

Fig. 5 Temperature-dependent temporal response. Temporal response of $I_{p}$ to periodic broadband light pulses, represented by shaded regions as examples for the first two switching cycles, where $I_{p}$ is plotted for $T \sim 100,200$, 300, and $350 \mathrm{~K} \mathrm{at} F \sim 10.75 \mathrm{~mW} / \mathrm{cm}^{2}$ for a $\mathrm{Gr}-$ perovskite and $\mathbf{b}$ Ag-perovskite PDs. The $\boldsymbol{\tau}_{\text {rise }}$ and $\boldsymbol{\tau}_{\text {fall }}$ as a function of $T$, where the $\boldsymbol{\tau}_{\text {rise }} \sim 31$ ms and $\boldsymbol{\tau}_{\text {fall }} \sim 27$ ms were measured for $\mathbf{c}$ Grperovskite PD, while for $\mathbf{d}$ Ag-perovskite PD, the $\boldsymbol{\tau}_{\text {rise }} \sim 17 \mathrm{~ms}$ and $\boldsymbol{\tau}_{\text {fall }} \sim 45 \mathrm{~ms}$ at $300 \mathrm{~K}$. At $T \sim 4 \mathrm{~K}$, the $\boldsymbol{\tau}_{\text {rise }}$ and $\boldsymbol{\tau}_{\text {fall }}$ values were found to be $\sim 59$ and $28 \mathrm{~ms}$, respectively, for Ag-perovskite PD.

Thorlabs DC2200 pulse modulator for the broadband light source, where the pulse width was fixed at $500 \mathrm{~ms}$. The rise and decay of $I_{p}$ is governed by trap states and recombination dynamics within the perovskite, and this temporal response thus serves as a gauge, to first order, to shed insights on the degree to which trap states influence photo-induced carrier dynamics. A good photosensitive material, such as a perovskite not only shows a high $I_{p}$ during the "ON" state, but it also yields a fast response time to the incoming radiation, as our measurements reveal. If the carrier dynamics are influenced by defects that serve as trapping centers, the response time is typically much slower ${ }^{53}$. In Fig. $5 a$, b, a fast increase and fast decay of $I_{p}$ were observed with good repeatability, followed by the saturation of $I_{p}$, where the Ag-perovskite PD demonstrated higher $I_{p}$ compared to the Gr-perovskite PD biased at $40 \mathrm{~V}$ over the entire temperature range tested. Figure $5 c$, d shows a more indepth analysis of the rise and decay time measurements obtained from our devices. The rise time $\boldsymbol{\tau}_{\text {rise }}$ and fall time $\boldsymbol{\tau}_{\text {fall }}$ are defined as the time it takes for the PD to reach $90 \%$ and drop to $10 \%$ of the maximum steady-state $I_{p}$, respectively ${ }^{54}$. At $\sim 300 \mathrm{~K}, \boldsymbol{\tau}_{\text {rise }} \sim 31 \mathrm{~ms}$ and $\boldsymbol{\tau}_{\text {fall }} \sim 27 \mathrm{~ms}$ were determined for the Gr-perovskite PD, while $\boldsymbol{\tau}_{\text {rise }} \sim 17 \mathrm{~ms}$ and $\boldsymbol{\tau}_{\text {fall }} \sim 45 \mathrm{~ms}$ were measured for the Ag-perovskite PD. Our data reveal that the Ag-perovskite PD is at least $8 X$ faster compared to some previous reports where evaporated Au served as the metal contact to $\mathrm{CH}_{3} \mathrm{NH}_{3} \mathrm{Pbl}_{3} \mathrm{PD}$ devices ${ }^{47}$. Table 1 summarizes the $\boldsymbol{\tau}_{\text {rise }}$ and $\boldsymbol{\tau}_{\text {fall }}$ of our inkjet-printed 2D perovskite PD devices contacted with solution-processed $\mathrm{Gr}$ and $\mathrm{Ag}$, along with a comparison of other figures of merits for PD to prior studies. While the fast photocurrent rise is attributed to the initial equilibration of photocarrier generation and recombination rates, the fast decay is due to the recombination of free carriers ${ }^{55}$. For both PDs, the $\boldsymbol{\tau}_{\text {rise }}$ and $\boldsymbol{\tau}_{\text {fall }}$ seem to decrease nonlinearly with increasing $T$, although the $\boldsymbol{\tau}_{\text {fall }}$ for the Ag-perovskite PD at $\sim 300 \mathrm{~K}$ seems to increase compared to the $\boldsymbol{\tau}_{\text {rise, }}$ which suggests that recombination of the photocarriers occurs over a longer duration at room $T$ for the Ag-perovskite. Generally, the values of $\boldsymbol{\tau}_{\text {rise }}$ and $\boldsymbol{\tau}_{\text {fall }}$ were lowest for $T \sim 100$ up to $150 \mathrm{~K}$ for both PDs, which is intriguing and may likely be associated with the orthorhombic-totetragonal crystal phase transition occurring in 2D perovskites within this temperature regime, as discussed previously ${ }^{39}$. The transients appear to be faster within this temperature range due possibly to the structural changes occurring within the $2 \mathrm{D}$ perovskite absorber layer, where carrier effective masses are likely to change from the crystalline phase transformation, which will influence switching speeds.

\section{Strain-dependent photoresponse of Ag-perovskite PD}

Strain engineering has been broadly applied to semiconducting materials, such as $\mathrm{Si}$ with the addition of $\mathrm{Ge}$, to achieve high switching speed transistors with both compressive and tensile strain. As the Ag-perovskite PD showed superior performance compared to the Gr-perovskite PDs, we then performed mechanical durability tests on the Ag-perovskite PD. The mechanical bending tests were carried out using 3D printed structures constructed at five different radii of curvatures: 0.072, 0.087, 0.112, 0.157 , and $0.262 \mathrm{~cm}^{-1}$. The experiment was performed under a 
(a)

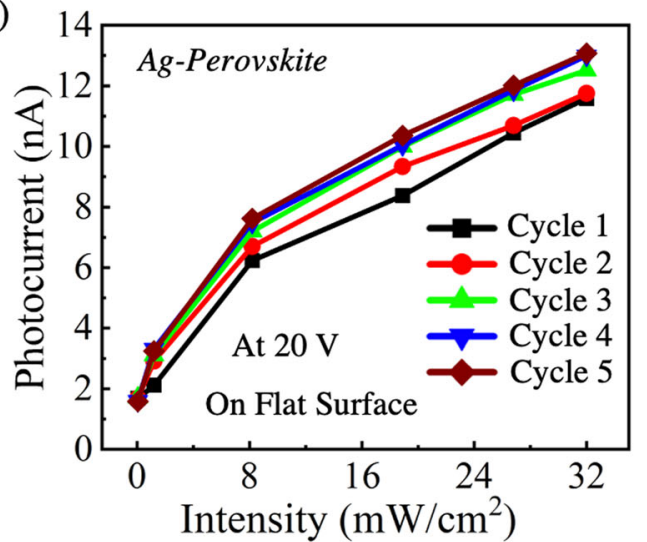

(b)

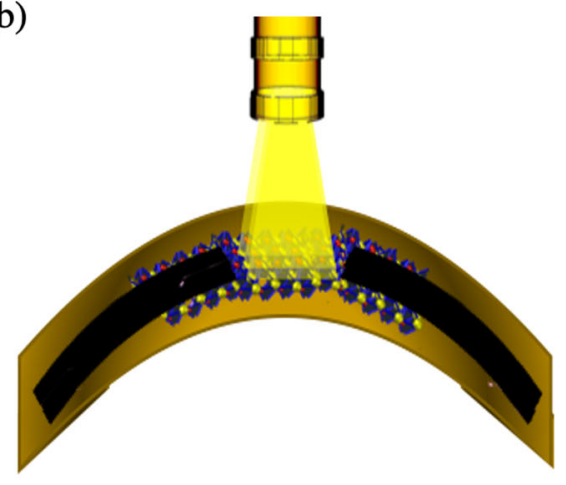

(c)

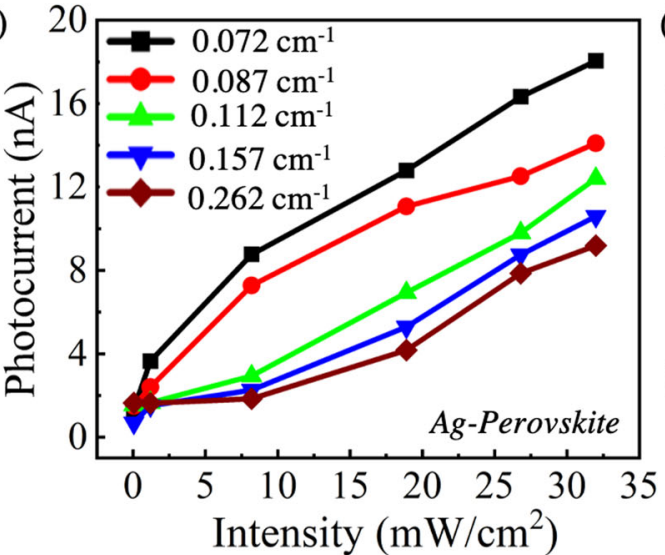

(d)

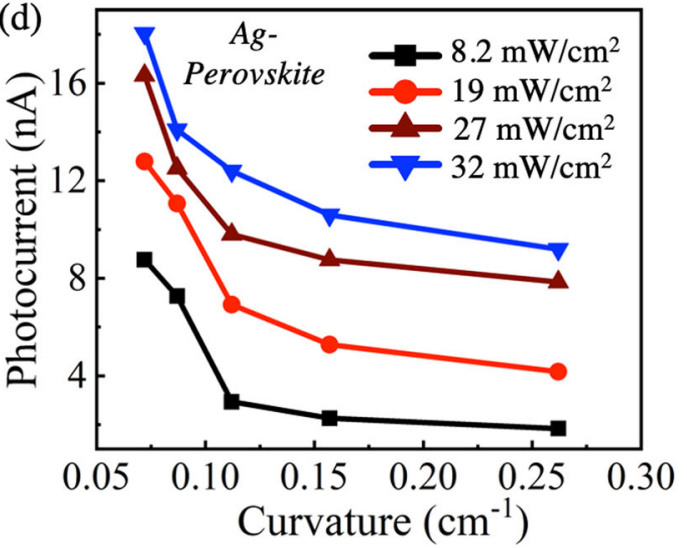

Fig. 6 Strain dependency. a Five repetitive cycles of the $I_{p}$ versus $F$, when the Ag-perovskite PD was on a flat surface. The $I_{p}$ did not change significantly for all the five cycles tested over the entire range of $F$ tested, which confirms the reproducibility of our inkjet-printed PD devices. b Schematic diagram of the strained Ag-perovskite PDs, while under illumination which was inkjet printed on the flexible PI substrate. c The change in $I_{p}$ as a function of $F$ for various strain/curvature levels: $\sim 0.072,0.087,0.112,0.157$, and $0.262 \mathrm{~cm}^{-1}$. d The dependency of $I_{p}$ as a function of curvature for various $F$, where the $I_{p}$ decreased as the curvature increased.

bias voltage of $\sim 20 \mathrm{~V}$, and the $I_{p}$ response was measured as a function of strain with varying light intensity. Figure 6 a displays the five repetitive cycles of $I_{p}$ measured as a function of $F$ on a flat surface. In the first cycle, $F$ was increased from $\sim 58 \mu \mathrm{W} / \mathrm{cm}^{2}$ up to the maximum of $32 \mathrm{~mW} / \mathrm{cm}^{2}$ with a time delay of $5 \mathrm{~s}$ in between each incremental $F$ to stabilize the response. The $F$ was then lowered from $\sim 32 \mathrm{~mW} / \mathrm{cm}^{2}$ back down to $\sim 58 \mu \mathrm{W} / \mathrm{cm}^{2}$, prior to initiating the second measurement cycle, all on a flat surface. As seen in Fig. $6 a$, there is no significant change in the $I_{p}$ at low $F \sim$ $58 \mu \mathrm{W} / \mathrm{cm}^{2}$ and $20 \mathrm{~V}$, while at higher $F$, the $I_{p}$ increased, likely due to electrical annealing of the contacts and the film, which requires further investigation in the future to better understand the underlying phenomenon. The increase in $I_{p}$ was tabulated to be $\sim 57 \%, 26 \%, 21 \%, 16 \%$, and $12 \%$ for $F \sim 1.2,8.2,18.9,26.8$, and $32 \mathrm{~mW} / \mathrm{cm}^{2}$, respectively, which indicates a gradual reduction in the net percent increase as $F$ increases.

Figure $6 \mathrm{~b}$ displays the schematic diagram of the strained flexible Ag-perovskite PD while under illumination. Figure $6 c$ shows the $I_{p}$ versus $F$ as a function of strain for all the five curvatures tested, where the increase in $I_{p}$ is consistent with increasing $F$ over all the curvatures. Similarly, the $I_{p}$ dependency with curvature at various $F$ is illustrated in Fig. $6 \mathrm{~d}$, and it is apparent the $I_{p}$ decreases with increasing strain levels over the range of $F$ used. The strain causes mechanical deformation and produces piezoelectric charges in the perovskite at the heterojunction interface. The electric field at the interface and the increased MS junction effective area provides a smaller driving force to separate the photogenerated electron-hole pairs, which reduces $I_{p}$. Also, Zhang et al. ${ }^{56}$ have discussed that the external strain also possibly changes the $\mathrm{Pb}-\mathrm{I}$ bond lengths, the $\mathrm{Pb}-\mathrm{I}-\mathrm{Pb}$ bond angles, and the orientations of the $\mathrm{CH}_{3} \mathrm{NH}_{3}{ }^{+}$molecules, and these strain-induced structural variations in the crystalline order result in reduced photocarrier generation. Their studies ${ }^{56}$ also shed insights on the photovoltaic properties of $\mathrm{CH}_{3} \mathrm{NH}_{3} \mathrm{Pbl}_{3}$ under external strain, where the absorption efficiency of incoming photons was lower.

In summary, we have synthesized $\left(\mathrm{CH}_{3}\left(\mathrm{CH}_{2}\right)_{3} \mathrm{NH}_{3}\right)_{2}\left(\mathrm{CH}_{3} \mathrm{NH}_{3}\right)_{n-1}$ $\mathrm{Pb}_{n} \mathrm{I}_{3 n+1}(n=4)$ family of $2 \mathrm{D}$ perovskites and characterized their properties using XRD (including at low-grazing angles) and SEM. Contact engineering of $2 \mathrm{D}$ layered perovskites is presented with $\mathrm{Gr}$ and $\mathrm{Ag}$ metals, where inkjet printing was used to fabricate heterostructure flexible PD devices on PI substrates. The $\Phi_{B}$ for our Gr-perovskite and Ag-perovskite PDs was extracted to be $\sim 920$ and $712 \mathrm{meV}$, respectively, in the $T$ regime from $\sim 100$ to $150 \mathrm{~K}$. The measured $R$ and $D$ for the Gr-perovskite and Ag-perovskite PDs confirmed the higher $R \sim 0.53 \mathrm{~A} / \mathrm{W}$ and $D \sim 3.24 \times 10^{13}$ Jones for the Ag-contacted 2D perovskite. Similarly, the EQE was found to be higher for Ag-perovskite when compared to Gr-perovskite PD. The ON/OFF ratio and response times, $\boldsymbol{\tau}_{\text {rise }}$ and $\boldsymbol{\tau}_{\text {fall }}$ for the Agperovskite PD were at least $30 \mathrm{X}$ and $8 \mathrm{X}$ higher, respectively, compared to prior reports. The $\lambda$-dependent $I_{p}$ measurement revealed that the maximum $I_{p}$ occurred at $\lambda \sim 630 \mathrm{~nm}$ for both types of devices, which is consistent with the energy bandgap of the 2D perovskite of our $n=4$ formulation. Lastly, straindependent bending tests showed the modulation of $I_{p}$ as a function of strain. The study presented here validates the 
importance of contacts to influence charge carrier dynamics, and our exceptional figures of merits particularly with inkjet-printed Ag contacts to $2 \mathrm{D}$ perovskites suggests that our solution-processed 2D perovskites are well positioned to have a significant impact on flexible optoelectronics and flexible PV research in the future.

\section{METHODS}

\section{Synthesis of the 2D perovskite crystal}

We synthesized the $\left(\mathrm{CH}_{3}\left(\mathrm{CH}_{2}\right)_{3} \mathrm{NH}_{3}\right)_{2}\left(\mathrm{CH}_{3} \mathrm{NH}_{3}\right)_{3} \mathrm{~Pb}_{4} \mathrm{l}_{13}$ perovskites, which is a family of layered compounds with tunable semiconducting properties. The PbO (part \#: 402982), $\mathrm{HI}$ (part \#: 210021), $\mathrm{H}_{3} \mathrm{PO}_{2}$ (part \#: 214906), $\mathrm{CH}_{3} \mathrm{NH}_{3} \mathrm{Cl}$ (part \#: 8060200250), and $n-\mathrm{CH}_{3}\left(\mathrm{CH}_{2}\right)_{3} \mathrm{NH}_{2}$ (part \#: 471305) were purchased from Sigma-Aldrich. The PbO powder $(2232 \mathrm{mg})$ was dissolved in a mixture of $57 \% \mathrm{w} / \mathrm{w}$ aqueous $\mathrm{HI}$ solution $(10.0 \mathrm{~mL})$ and $50 \%$ aqueous $\mathrm{H}_{3} \mathrm{PO}_{2}(1.7 \mathrm{~mL})$ by heating at $130^{\circ} \mathrm{C}$ and subsequent boiling under constant magnetic stirring of $350 \mathrm{rpm}$ for about $25 \mathrm{~min}$, that led to the formation of a bright yellow solution. Subsequent addition of solid $\mathrm{CH}_{3} \mathrm{NH}_{3} \mathrm{I}$ (507 mg) to the hot yellow solution initially caused the precipitation of a black powder that rapidly redissolved under stirring to afford a clear bright yellow solution. Additionally, the $n-\mathrm{CH}_{3}\left(\mathrm{CH}_{2}\right)_{3} \mathrm{NH}_{3} \mathrm{I}$ $(248 \mu \mathrm{L})$ and hydriodic acid $(5 \mathrm{~mL})$ were mixed separately in a glass vial and the mixture was then added dropwise to the solution under vigorous stirring for $10 \mathrm{~min}$, which did not result in any visible changes to the solution. After $10 \mathrm{~min}$, we stopped stirring and heating, which were previously at $350 \mathrm{rpm}$ and $130{ }^{\circ} \mathrm{C}$, respectively. The solution was left to cool to room temperature during which time deep black, rectangular-shaped platelets started to crystallize. The precipitation was deemed to be complete after $\sim 2 \mathrm{~h}$. The crystals were isolated by suction filtration and thoroughly dried under reduced pressure ${ }^{21}$.

\section{D perovskite ink formulation}

Perovskite inks were formed using a magnetically stirred solvent engineering process. The $30 \mathrm{mg} / \mathrm{mL}$ of $\left(\mathrm{CH}_{3}\left(\mathrm{CH}_{2}\right)_{3} \mathrm{NH}_{3}\right)_{2}\left(\mathrm{CH}_{3} \mathrm{NH}_{3}\right)_{3} \mathrm{~Pb}_{4} \mathrm{I}_{13}$ powder was dissolved in $10 \mathrm{~mL}$ of DMF and magnetically stirred overnight at $500 \mathrm{rpm}$ at room $T$, as shown in Fig. 1a-left. Then the solution was kept at room $T$ for 30 min to stabilize the solution for inkjet printing. The solubility of the perovskite precursor in DMF is excellent, as DFM is less volatile and hygroscopic in nature compared to other typical solvents used in inkjet printing, such as $\mathrm{N}$-methylpyrrolidon. Using DFM as the solvent for perovskite inks has previously been reported ${ }^{57}$, which guided us to adopt this for our ink formulations. The top $3 \mathrm{~mL}$ of the perovskite ink was then transferred to the cartridge of the Dimatix Materials Printer (DMP-2850 Series) for our subsequent device fabrication efforts using inkjet printing.

\section{Gr and $\mathrm{Ag}$ ink formulations}

Both Gr (SKU \# 496596) and Ag (SKU \# 327085) powders were purchased from Sigma-Aldrich, which had a purity of $\geq 99.99 \%$ for trace metals, while the particle size was $\sim 45$ and $2-3.5 \mu \mathrm{m}$ as received, for the two materials, respectively. Additionally, the liquid exfoliated $\mathrm{Gr}$ ink was characterized using Raman spectroscopy where three distinct Raman peaks were observed at $1361 \mathrm{~cm}^{-1}$ (D-band), at $1580 \mathrm{~cm}^{-1}$ (G-band), and at $2713 \mathrm{~cm}^{-1}$ (2D-band). The $D$-band indicates the defective structure of exfoliated graphite sheets, while the $G$ - and 2D-bands indicate the order and purity of the characteristic graphite/graphene sheets, respectively ${ }^{58}$. Raman Spectroscopy has previously been used to tabulate the $I_{D} / I_{G}$ and $I_{2 D} / I_{G}$ ratios, where the latter is correlated to thickness. For example, Nguyen et al. ${ }^{58}$ notes that when the ratio $I_{2 D} / l_{G} \sim 2-3$, it is reflective of monolayer graphene, when $2>I_{2 \mathrm{D}} / I_{G}>1$, bilayer graphene results, and finally for multilayer graphene, the $I_{2 D} / I_{G}<1$. Our liquid exfoliated graphene showed ratios for $I_{D} / I_{G} \sim 0.04$ and $I_{2 D} / l_{G} \sim 0.28$, where the latter clearly indicates that our liquid exfoliated graphene is multilayer ${ }^{58}$. The conducting inks for the electrodes were produced through ultrasonic bath sonication of $\mathrm{Gr}$ or Ag powder in 2-propanol (IPA) as shown in Fig. 1aright. An initial concentration of ethyl cellulose of $\sim 0.8 \mathrm{wt} \%$ and $50 \mathrm{mg} / \mathrm{ml}$ of $\mathrm{Gr}$ or Ag powder was then added, respectively, in $10 \mathrm{ml}$ of IPA and placed in a Branson Bath Sonicator (CPX2800) for $24 \mathrm{~h}$. The top $8 \mathrm{ml}$ of the supernatant was removed and placed in a new vial, which was later transferred to the cartridge of the inkjet printer. The formulation of the $\mathrm{Gr}$ or Ag with IPA is challenging compared to the other inks, such as $\mathrm{MoS}_{2}$ ink with Cyclohexanone/Terpineol solvent ${ }^{59}$, because the IPA-based inks frequently clog the nozzles; the IPA solvent dries out fast and makes the $\mathrm{Gr} / \mathrm{Ag}$ platelets to compromise the performance of the nozzle head, so frequent nozzle cleaning is necessary, as reflected in Fig. 1b. For nozzle cleaning, the cartridge head was placed in a small glass beaker filled with IPA, which was then sonicated for $2 \mathrm{~min}$ to remove the nozzle clogs.

\section{Inkjet-printed heterostructure PD fabrication}

A $2 \times 1.5 \mathrm{~mm}$ perovskite layer was first printed with 30 ink passes on a flexible PI substrate. Printing the perovskite ink is challenging compared to other 2D printed materials such as $\mathrm{MoS}_{2}$ or graphene inks ${ }^{59,60}$. Solubility is one issue even after the printed layer becomes solid, since the subsequent layer can dissolve the initial layer, as the addition of the newly printed ink makes it soluble again. That is why a stable base layer is required so that the other printed layers can stay confined within the printed area. To make a stable base layer, four nozzles were used to print five passes and allowed to stabilize for $15 \mathrm{~min}$ to alleviate the surface tension between the printed ink and the flexible PI substrate. Then, the rest of the 25 passes were printed. The printing parameters, such as drop spacing, were kept at $30 \mu \mathrm{m}$, platen $T \sim 40^{\circ} \mathrm{C}$, the jetting voltage $\sim 21 \mathrm{~V}$, and these parameters have been optimized after many iterations. The printed perovskite was annealed at $30^{\circ} \mathrm{C}$ for $30 \mathrm{~min}$ on a hot plate, where the $T$ was ramped to $100^{\circ} \mathrm{C}$ ( $T$ ramp rate $\sim 30^{\circ} \mathrm{C} / \mathrm{min}$ ) and, subsequently, the film was annealed for $30 \mathrm{~min}$. Thermal annealing is a crucial step to accelerate the reaction between the organic and inorganic species in the perovskite compound. Dualeh et al. ${ }^{61}$ established that densely interconnected films are obtained by annealing the perovskite at $80^{\circ} \mathrm{C}$ for $3 \mathrm{~h}$, or $100^{\circ} \mathrm{C}$ for $45 \mathrm{~min}$, respectively. When the $T$ is lower than $80^{\circ} \mathrm{C}$, the perovskite phase formation appeared to be hindered. Furthermore, prolonging the annealing time at low- $T$ does not provide any advantage for film quality. On the other hand, the perovskite films annealed at $T$ more than $100^{\circ} \mathrm{C}$ will produce secondary phases of $\mathrm{Pbl}_{2}$, likely originating from the decomposition of $\mathrm{MAPbl}_{3}{ }^{61}$. Using excessively high or low processing $T$ will thus cause undesired film quality, as a result of slow reaction rates or additional side/ decomposition reactions.

After annealing the perovskite layer, the PI substrate was placed back onto the platen at the same $(x, y)$ coordinates for printing the Gr/Ag contacts, where $\sim 25$ ink passes were used, and the films were annealed at $100^{\circ} \mathrm{C}$ for $30 \mathrm{~min}$. The separation between the metal electrodes was $\sim 800 \mu \mathrm{m}$ and the width of each electrode was $\sim 600 \mu \mathrm{m}$, as the contact layers were printed on top of the perovskite layer.

\section{DATA AVAILABILITY}

The data that support the findings of this study are available upon the request from the corresponding author.

Received: 16 October 2020; Accepted: 16 February 2021; Published online: 25 March 2021

\section{REFERENCES}

1. Zhou, H. et al. Interface engineering of highly efficient perovskite solar cells. Science 345, 542-546 (2014).

2. Green, M. A., Ho-Baillie, A. \& Snaith, H. J. The emergence of perovskite solar cells. Nat. Photon. 8, 506 (2014).

3. Boix, P. P., Agarwala, S., Koh, T. M., Mathews, N. \& Mhaisalkar, S. G. Perovskite solar cells: beyond methylammonium lead iodide. J. Phys. Chem. Lett. 6, 898-907 (2015).

4. Guo, Y., Liu, C., Tanaka, H. \& Nakamura, E. Air-stable and solution-processable perovskite photodetectors for solar-blind UV and visible light. J. Phys. Chem. Lett. 6, 535-539 (2015).

5. Zhang, Y. et al. Synthesis, properties, and optical applications of low-dimensional perovskites. Chem. Commun. 52, 13637-13655 (2016).

6. Liu, J. et al. Two-dimensional $\mathrm{CH} 3 \mathrm{NH} 3 \mathrm{Pbl} 3$ perovskite: synthesis and optoelectronic application. ACS Nano 10, 3536-3542 (2016).

7. Qi, X. et al. Photonics and optoelectronics of 2D metal-halide perovskites. Small 14, 1800682 (2018).

8. Stranks, S. D. et al. Electron-hole diffusion lengths exceeding 1 micrometer in an organometal trihalide perovskite absorber. Science. 342, 341-344 (2013).

9. Kojima, A., Teshima, K., Miyasaka, T. \& Shirai, Y. Novel photoelectrochemical cell with mesoscopic electrodes sensitized by lead-halide compounds (2). In: Meeting Abstracts, Vol. 397 (The Electrochemical Society, 2006). 
10. Green, M., Dunlop, E., Hohl-Ebinger, J., Yoshita, M., Kopidakis, N. \& Hao, X. Solar cell efficiency tables (version 57). Prog. Photovolt. Res. Appl. 29, 3-15 (2021).

11. Kim, Y. et al. Multicolored organic/inorganic hybrid perovskite light-emitting diodes. Adv. Mater. 27, 1248-1254 (2015).

12. Dou, L. et al. Solution-processed hybrid perovskite photodetectors with high detectivity. Nat. Commun. 5, 1-6 (2014).

13. Lu, H. et al. A self-powered and stable all-perovskite photodetector-solar cell nanosystem. Adv. Funct. Mater. 26, 1296-1302 (2016).

14. Xie, C. \& Yan, F. Flexible photodetectors based on novel functional materials. Small 13, 1701822 (2017).

15. Konstantatos, G. Current status and technological prospect of photodetectors based on two-dimensional materials. Nat. Commun. 9, 1-3 (2018).

16. Kim, J. et al. Near-ultraviolet-sensitive graphene/porous silicon photodetectors. ACS Appl. Mater. Interfaces 6, 20880-20886 (2014).

17. Haugan, H. J. et al. Study of residual background carriers in midinfrared In As/Ga $\mathrm{Sb}$ superlattices for uncooled detector operation. Appl Phys. Lett. 92, 71102 (2008).

18. Jin, Y., Wang, J., Sun, B., Blakesley, J. C. \& Greenham, N. C. Solution-processed ultraviolet photodetectors based on colloidal $\mathrm{ZnO}$ nanoparticles. Nano Lett. 8 1649-1653 (2008).

19. Ruddlesden, S. N. \& Popper, P. New compounds of the K2NiF4 type. Acta Crystallogr. 10, 538-539 (1957).

20. Birowosuto, M. D. et al. X-ray scintillation in lead halide perovskite crystals. Sci. Rep. 6, 37254 (2016).

21. Stoumpos, C. C. et al. Ruddlesden-Popper hybrid lead iodide perovskite 2D homologous semiconductors. Chem. Mater. 28, 2852-2867 (2016).

22. Min, M., Hossain, R. F., Adhikari, N. \& Kaul, A. B. Inkjet-printed organohalide 2D layered perovskites for high-speed photodetectors on flexible polyimide substrates. ACS Appl. Mater. Interfaces 12, 10809-10819 (2020).

23. Yuan, Z., Shu, Y., Xin, Y. \& Ma, B. Highly luminescent nanoscale quasi-2D layered lead bromide perovskites with tunable emissions. Chem. Commun. 52, 3887-3890 (2016).

24. Aharon, S. \& Etgar, L. Two dimensional organometal halide perovskite nanorods with tunable optical properties. Nano Lett. 16, 3230-3235 (2016).

25. Sze, S. M. \& Ng, K. K. Physics of Semiconductor Devices (John Wiley \& Sons, 2006).

26. Fan, Z. -Q. et al. In-plane Schottky-barrier field-effect transistors based on $1 \mathrm{~T} / 2 \mathrm{H}$ heterojunctions of transition-metal dichalcogenides. Phys. Rev. B 96, 165402 (2017).

27. Bandyopadhyay, A. S., Adhikari, N. \& Kaul, A. B. Quantum Multibody Interactions in Halide-assisted vapor-synthesized monolayer WSe2 and its integration in a high responsivity photodetector with low-interface trap density. Chem. Mater. 31, 9861-9874 (2019).

28. Michel, M. et al. A thermally-invariant, additively manufactured, high-power graphene resistor for flexible electronics. 2D Mater. 4, 25076 (2017).

29. Jayanand, K. et al. Sc 3 N@ C 80 and La@ C 82 doped graphene for a new class of optoelectronic devices. J. Mater. Chem. C 8, 3970-3981 (2020).

30. Hossain, R. F., Deaguero, I. G., Boland, T. \& Kaul, A. B. Solution dispersed 2D graphene \& MoS2 for an inkjet printed biocompatible photodetector. In: 2016 Lester Eastman Conference (LEC), Vol. 2016, 19-22 (IEEE, 2016).

31. Lin, C. - H. et al. Metal contact and carrier transport in single crystalline $\mathrm{CH} 3 \mathrm{NH} 3 \mathrm{PbBr} 3$ perovskite. Nano Energy 53, 817-827 (2018).

32. Guo, Y., Saidi, W. A. \& Wang, Q. 2D halide perovskite-based van der Waals heterostructures: contact evaluation and performance modulation. 2D Mater. 4 35009 (2017)

33. Kim, N. -K. et al. Investigation of thermally induced degradation in $\mathrm{CH} 3 \mathrm{NH} 3 \mathrm{~Pb}$ 3 perovskite solar cells using in-situ synchrotron radiation analysis. Sci. Rep. 7, 1-9 (2017).

34. Zhang, Y. et al. Efficient inverted planar formamidinium lead iodide perovskite solar cells via a post improved perovskite layer. RSC Adv. 6, 79952-79957 (2016).

35. Gangadharan, D. T. \& Ma, D. Searching for stability at lower dimensions: current trends and future prospects of layered perovskite solar cells. Energy Environ. Sci. 12, 2860-2889 (2019).

36. Eaton, S. W. et al. Lasing in robust cesium lead halide perovskite nanowires. Proc Natl Acad. Sci. 113, 1993-1998 (2016).

37. Liu, X. -D. et al. Solution-phase growth of organolead halide perovskite nanowires and nanoplates assisted by long-chain alkylammonium and solvent polarity. Mater. Lett. 206, 75-79 (2017).

38. Wang, Q. et al. Large fill-factor bilayer iodine perovskite solar cells fabricated by a low-temperature solution-process. Energy Environ. Sci. 7, 2359-2365 (2014).

39. Weller, M. T., Weber, O. J., Henry, P. F., Di Pumpo, A. M. \& Hansen, T. C. Complete structure and cation orientation in the perovskite photovoltaic methylammonium lead iodide between 100 and 352 K. Chem. Commun. 51, 4180-4183 (2015).

40. Anwar, A., Nabet, B., Culp, J. \& Castro, F. Effects of electron confinement on thermionic emission current in a modulation doped heterostructure. J. Appl Phys. 85, 2663-2666 (1999).
41. Chen, Y. -F. et al. Evidence of band bending induced by hole trapping at MAPbI 3 perovskite/metal interface. J. Mater. Chem. A 4, 17529-17536 (2016).

42. $\mathrm{Hu}, \mathrm{X}$. et al. High-performance flexible broadband photodetector based on organolead halide perovskite. Adv. Funct. Mater. 24, 7373-7380 (2014).

43. Wang, W., Ma, Y. \& Qi, L. High-performance photodetectors based on organometal halide perovskite nanonets. Adv. Funct. Mater. 27, 1603653 (2017).

44. Li, P., Shivananju, B. N., Zhang, Y., Li, S. \& Bao, Q. High performance photodetector based on 2D CH3NH3Pbl3 perovskite nanosheets. J. Phys. D Appl Phys. 50, 94002 (2017).

45. Deng, $H$. et al. Flexible and semitransparent organolead triiodide perovskite network photodetector arrays with high stability. Nano Lett. 15, 7963-7969 (2015).

46. Maculan, $\mathrm{G}$. et al. $\mathrm{CH} 3 \mathrm{NH} 3 \mathrm{PbCl} 3$ single crystals: inverse temperature crystallization and visible-blind UV-photodetector. J. Phys. Chem. Lett. 6, 3781-3786 (2015).

47. Zhang, X. et al. High-switching-ratio photodetectors based on perovskite CH3NH3Pbl3 nanowires. Nanomaterials 8, 318 (2018).

48. Yao, J. D., Zheng, Z. Q., Shao, J. M. \& Yang, G. W. Stable, highly-responsive and broadband photodetection based on large-area multilayered WS 2 films grown by pulsed-laser deposition. Nanoscale 7, 14974-14981 (2015).

49. Riazimehr, S. et al. High responsivity and quantum efficiency of graphene/silicon photodiodes achieved by interdigitating Schottky and gated regions. ACS Photonics 6, 107-115 (2018).

50. Ulaganathan, R. K. et al. High photosensitivity and broad spectral response of multi-layered germanium sulfide transistors. Nanoscale 8, 2284-2292 (2016).

51. Lv, L. et al. Generalized colloidal synthesis of high-quality, two-dimensional cesium lead halide perovskite nanosheets and their applications in photodetectors. Nanoscale 8, 13589-13596 (2016).

52. Zhang, M. et al. High-performance photodiode-type photodetectors based on polycrystalline formamidinium lead iodide perovskite thin films. Sci. Rep. 8, 1-9 (2018).

53. Law, J. B. K. \& Thong, J. T. L. Simple fabrication of a $\mathrm{ZnO}$ nanowire photodetector with a fast photoresponse time. Appl Phys. Lett. 88, 133114 (2006).

54. Wang, Y. et al. High sensitivity and fast response solution processed polymer photodetectors with polyethylenimine ethoxylated (PEIE) modified ITO electrode. Opt. Express 25, 7719-7729 (2017).

55. Cunningham, G. et al. Photoconductivity of solution-processed MoS 2 films. J. Mater. Chem. C 1, 6899-6904 (2013).

56. Zhang, L. et al. Strain induced electronic structure variation in methyl-ammonium lead iodide perovskite. Sci. Rep. 8, 1-9 (2018).

57. Gu, Z., Huang, Z., Li, C., Li, M. \& Song, Y. A general printing approach for scalable growth of perovskite single-crystal films. Sci. Adv. 4, eaat2390 (2018).

58. Nguyen, V. T. et al. Synthesis of multi-layer graphene films on copper tape by atmospheric pressure chemical vapor deposition method. Adv. Nat. Sci. Nanosci. Nanotechnol. 4, 35012 (2013).

59. Hossain, R. F., Deaquero, I. G., Boland, T. \& Kaul, A. B. Biocompatible, large-format, inkjet printed heterostructure MoS 2-graphene photodetectors on conformable substrates. npj 2D Mater. Appl. 1, 1-10 (2017).

60. Fadil, D., Hossain, R. F., Saenz, G. A. \& Kaul, A. B. On the chemically-assisted excitonic enhancement in environmentally-friendly solution dispersions of twodimensional MoS2 and WS2. J. Mater. Chem. C 5, 5323-5333 (2017).

61. Dualeh, A. et al. Impedance spectroscopic analysis of lead iodide perovskitesensitized solid-state solar cells. ACS Nano. 8, 362-373 (2014).

\section{ACKNOWLEDGEMENTS}

We thank the Office of Naval Research (Grant Number ONR N00014-20-1-2597) that enabled us to pursue this work. A.B.K. is also grateful to the support from the PACCAR Technology Institute at UNT and the Endowed Professorship support.

\section{AUTHOR CONTRIBUTIONS}

A.B.K. conceived the overall project. R.F.H. conducted the experiments related to ink formulation, inkjet printing, material characterization, and device measurements. M.M. developed techniques to synthesize the 2D perovskites. L.-C.M. and S.R.S. contributed to discussions related to figure edits. A.B.K and R.F.H. analyzed the data, conducted significant paper edits, and came up with the conclusions. All reviewed the paper writing and approved of its content.

\section{COMPETING INTERESTS}

The authors declare no competing interests. 


\section{ADDITIONAL INFORMATION}

Correspondence and requests for materials should be addressed to A.B.K.

Reprints and permission information is available at http://www.nature.com/reprints

Publisher's note Springer Nature remains neutral with regard to jurisdictional claims in published maps and institutional affiliations.

Open Access This article is licensed under a Creative Commons Attribution 4.0 International License, which permits use, sharing, adaptation, distribution and reproduction in any medium or format, as long as you give appropriate credit to the original author(s) and the source, provide a link to the Creative Commons license, and indicate if changes were made. The images or other third party material in this article are included in the article's Creative Commons license, unless indicated otherwise in a credit line to the material. If material is not included in the article's Creative Commons license and your intended use is not permitted by statutory regulation or exceeds the permitted use, you will need to obtain permission directly from the copyright holder. To view a copy of this license, visit http://creativecommons. org/licenses/by/4.0/.

(c) The Author(s) 2021 